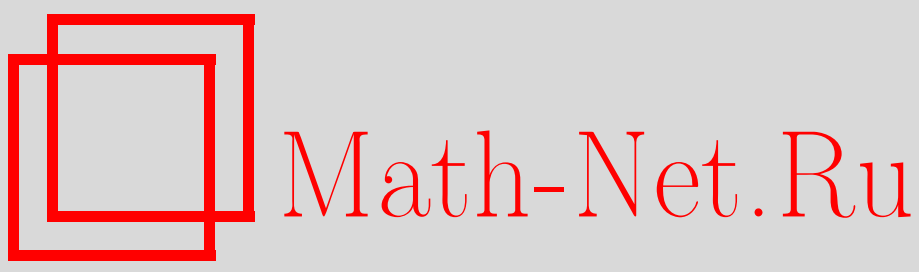

А. Е. Шишков, А. Г. Щелков, Граничные режимы с обострением для общих квазилинейных параболических уравнений в многомерных областях, Матем. сб., 1999, том 190, номер 3, 129-160

DOI: https://doi.org/10.4213/sm398

Использование Общероссийского математического портала Math-Net.Ru подразумевает, что вы прочитали и согласны с пользовательским соглашением

http://www.mathnet.ru/rus/agreement

Параметры загрузки:

IP: 54.80 .97 .219

26 апреля 2023 г., 17:56:00 


\author{
А.Е.Шишков, А.Г. Шелков
}

\title{
Граничные режимы с обострением для общих квазилинейных параболических уравнений в многомерных областях
}

\begin{abstract}
Предлагается новый, не опирающийся на барьерную технику, подход к изучению асимптотических свойств обобщенных решений параболических начально-краевых задач с обострением граничных данных в конечный момент времени. Устанавливаются точные условия на характер обострения, обеспечивающие равномерную локализацию решения при произвольной финитной начальной функции. Основной результат статьи - это получение точных достаточных условий того, что множество сингулярности (или множество blow-up) произвольного решения остается сосредоточенным на границе области.
\end{abstract}

Библиография: 19 названий.

\section{$\S 1$. Введение}

В работе изучаются некоторые качественные свойства обобщенных решений смешанной задачи Коши-Дирихле для широкого класса квазилинейных дивергентных вырождаюшихся параболических уравнений типа нестационарной нњютоновско-неньютоновской фильтрации. Природу этих свойств продемонстрируем на примере следуюшей начально-краевой задачи для модельного уравнения нелинейной теплопроводности:

$$
\begin{gathered}
u_{t}=\left(u^{m}\right)_{x x} \quad \forall(t, x) \in(0, T) \times(0, \infty), \quad m>1, \quad T<\infty ; \\
u(0, x)=u_{0}(x) \quad \forall x \in[0, \infty), \\
u(t, 0)=f(t) \quad \forall t \in[0, T), \\
f(t) \rightarrow \infty \text { при } t \rightarrow T,
\end{gathered}
$$

где $u_{0}$ и $f$ являются заданными неотрицательными непрерывньми функциями, удовлетворяюшими следуюшему условию согласования: $u_{0}(0)=f(0)$. В случае ограниченности $u_{0}$ указанная задача имеет, как хорошо известно [1], единственное обобшенное решение $u(t, x)$. Это решение является непрерывной функцией [2], [3], причем если $u_{0}$ имеет компактный носитель, то для всех $t \in[0, T)$ носитель $u(t, \cdot)$ компактен (см. [4]-[6]) и можно определить подвижную границу (иначеговоря, термальный фронт, см. [7]):

$$
\zeta(t)=\sup \{x \in[0, \infty): u(t, x)>0\} \quad \forall t \in[0, T) .
$$

Работа вьполнена при частичной поддержке фонда INTAS (грант № 96-1060). 
Эта функция является непрерывной и монотонно возрастающей [8]. Условие (1.4) определяет так называемый граничный режим с обострением со временем обострения $T$. Наличие такого режима приводит к необходимости изучения предельного поведения решения $u(t, x)$ и фронта $\zeta(t)$ при $t \rightarrow T$. Такое изучение проводилось во многих исследованиях (см. [9], [10] и указанную там литературу).

ОПРЕДЕЛЕниЕ 1.1. Задача (1.1)-(1.3) при граничном режиме с обострением (1.4) обладает свойством локализации, если

$$
\lim _{t \rightarrow T} \zeta(t)<\infty
$$

ОПРЕДЕЛЕНИЕ 1.2. Множество $\Omega \in[0, \infty)$ называется множеством интенсификащии или множсеством сингулярности решения $u(t, x)$ задачи (1.1)-(1.4), если для любой точки $x \in \Omega$

$$
\limsup _{t \rightarrow T} u(t, x)=\infty
$$

Нахождение точных условий локализации решений и описание структуры множества сингулярности являются основными вопросами в проблематике, связанной с неограниченными граничными режимами. Первый общий результат в этом направлении был получен в [11]. В предположении, что

$$
\begin{gathered}
f(t) \in C^{2}(0, T), \quad f^{\prime}(t)>0 \forall t \in(0, T), \\
\text { существует } \lim _{t \rightarrow T}\left(\frac{f(t)}{f^{\prime}(t)}\right)^{\prime}=l,
\end{gathered}
$$

было доказано, что произвольное решение $u(t, x)$ задачи $(1.1)-(1.4)$ сходится к некоторому аппроксимационному автомодельному решению при $t \rightarrow T$ равномерно по всем $x \in[0, \infty)$. Форма этого автомодельного решения позволяет сделать заключение, что локализация имеет место в том и только том случае, если

$$
l=-\infty \text { или } \frac{f(t)^{m}}{f^{\prime}(t)} \in L_{\infty}(0, T),
$$

причем справедлива следующая характеристика множества сингулярности $\Omega$ :

$$
\omega \equiv \sup \{x: x \in \Omega\}= \begin{cases}0, & \text { если } l=-\infty, \\ c \limsup _{t \rightarrow T}\left[\frac{f(t)^{m}}{f^{\prime}(t)}\right]^{1 / 2}, & \text { если } l>-\infty\end{cases}
$$

где $c$ - постоянная, зависяшая лишп от $l$.

Ранее [12] этот результат был установлен для модельного, предельного с точки зрения условия (1.9) граничного режима

$$
f(t)=k(T-t)^{-\frac{1}{m-1}}, \quad 0<k=\text { const }<\infty .
$$


В книге [10] рассмотрены граничные режимы с обострением и для некоторых других уравнений нелинейной теплопроводности. Так, для уравнений с градиентной нелинейностью

$$
u_{t}=\left(\left|u_{x}\right|^{p-1} u_{x}\right)_{x}
$$

найдено автомодельное решение, соответствуюшее граничной функции

$$
f(t)=k(T-t)^{-n}, \quad n>0 .
$$

Из структуры этого решения следует, что предельньй граничный режим, не разрушающий свойство локализации ( $S$-режим в терминологии [10]), соответствует значению $n=(p-1)^{-1}, p>1$.

Ослабление жестких условий (1.8), (1.7) было осуществлено в [9]. Установлено, что для локализации необходимо и достаточно, чтобы

$$
\limsup _{t \rightarrow T}\left[\frac{1}{f(t)} \int_{0}^{t} f(s)^{m} d s\right]<\infty
$$

при этом если имеет место локализация, то

$$
\limsup _{t \rightarrow T}(T-t)^{\frac{1}{m-1}} u(t, x)<\infty \quad \forall x>0,
$$

если же локализация не имеет места, то

$$
\limsup _{t \rightarrow T}(T-t)^{\frac{1}{m-1}} u(t, x)=\infty \quad \forall x \geqslant 0
$$

кроме того,

$$
\liminf _{t \rightarrow T} u(t, x)=\infty \quad \forall x \in \Omega
$$

и множество сингулярности $\Omega$ является связным интервалом $[0, \omega)$. Получена также обшая двусторонняя оценка $\omega$, из которой вытекает, в частности,

$$
\nu b^{\frac{m-1}{2}} \leqslant \omega \leqslant \nu B^{\frac{m-1}{2}},
$$

где

$$
\begin{gathered}
\nu=\left[\frac{2 m(m+1)}{m-1}\right]^{1 / 2}, \quad B=\limsup _{t \rightarrow T}(T-t)^{\frac{1}{m-1}} f(t), \\
b=\liminf _{t \rightarrow T}(T-t)^{\frac{1}{m-1}} f(t) .
\end{gathered}
$$

Показано также, что при выполнении условий (1.7), (1.8)

$$
\limsup _{t \rightarrow T}\left[\frac{1}{f(t)} \int_{0}^{t} f(s)^{m} d s\right]=\limsup _{t \rightarrow T} \frac{f(t)^{m}}{f^{\prime}(t)} .
$$


Обобщение указанных результатов из [11] для задачи Неймана с граничным режимом с обострением для уравнения (1.1) было проведено в [13].

Отметим теперь, что все имеющиеся результаты по граничньм режимам с обострением получены барьерной техникой и связаны с нахождением различных явных автомодельных решений. Этот подход в принципе неприменим к уравнениям, не допускающим соответствующих теорем сравнения.

В настоящей работе предлагается другой, не связанный с барьерной техникой подход к указанным задачам, позволяющий рассмотреть широкий класс квазилинейных параболических уравнений в многомерных областях. Устанавливаются точные достаточные условия локализации решения, точные условия локализации множества сингулярности решения на границе исходной области.

Наш метод основан на специальных интегральных априорных оценках решений рассматриваемых задач и объединяет идеи, развивавшиеся в рамках:

а) метода локальных энергетических оценок (см. [14], [15]);

б) метода априорных оценок типа принципа Сен-Венана в теории единственности обобшенных решений линейных параболических задач (см. [16]);

в) нелинейного варианта метода оценок типа принципа Сен-Венана, предложенного в [17].

\section{§ 2. Формулировка основных результатов}

В области $Q=(0, T) \times \Omega, \Omega=\Omega_{R} \equiv\left\{x \in \mathbb{R}^{n}: 1<|x|<R\right\}, n \geqslant 1,0<T<\infty$, $R<\infty$, рассматривается следуюшая задача Коши-Дирихле:

$$
\begin{gathered}
\frac{\partial}{\partial t}\left(|u|^{q-1} u\right)-\sum_{i=1}^{n} D_{x_{i}} a_{i}\left(t, x, u, D_{x} u\right)=0, \quad q>0 ; \\
\left.u\right|_{\Gamma(1)}=\tilde{f}(t, x),\left.\quad u\right|_{\Gamma(R)}=0 ; \\
u(0, x)=u_{0} \in L_{q+1}(\Omega) ; \\
\operatorname{supp} u_{0} \in\left\{x \in \mathbb{R}^{n}:|x|<d\right\}, \quad 1<d<R .
\end{gathered}
$$

Здесь $\Gamma(s) \equiv(0, T) \times \partial B(s), B(s)=\left\{x \in \mathbb{R}^{n}:|x|<s\right\}$, каратеодориевы функции $a_{i}$ удовлетворяют следующим условиям коэрцитивности и роста:

$$
\begin{array}{r}
\sum_{i=1}^{n} a_{i}(t, x, s, \xi) \xi_{i} \geqslant d_{0}|\xi|^{p+1} \quad \forall(t, x, s, \xi) \in \bar{Q} \times \mathbb{R}^{1} \times \mathbb{R}^{n}, \quad d_{0}>0 ; \\
\left|a_{i}(t, x, s, \xi)\right| \leqslant d_{1}|\xi|^{p} \quad \forall(t, x, s, \xi) \in \bar{Q} \times \mathbb{R}^{1} \times \mathbb{R}^{n}, d_{1}<\infty, i=1,2, \ldots, n .
\end{array}
$$

Вьполняется основное структурное условие:

$$
0<q<p
$$

т.е. уравнение (2.1) является уравнением типа "медленной" диффузии. Граничная функция $\tilde{f}$ является следом на $\Gamma(1)$ некоторой функции $f(t, x)$ такой, что

$$
\begin{gathered}
f \in L_{\infty}\left(\left(0, T_{0}\right) \times \Omega\right) \cap L_{p+1}\left(0, T_{0} ; W_{p+1}^{1}(\Omega)\right) \quad \forall T_{0}<T, \\
\operatorname{supp} f \in[0, T] \times B\left(R_{0}\right), \quad R_{0}<R, \\
f_{t}^{\prime} \in L_{1}\left(0, T_{0} ; L_{q+1}(\Omega)\right) \cap L_{\frac{p+1}{p-q+1}}\left(0, T_{0} ; L_{\frac{p+1}{p-q+1}}(\Omega)\right) \quad \forall T_{0}<T .
\end{gathered}
$$


Характер обострения граничного режима при $t=T$ будем описывать функцией

$$
\begin{aligned}
F(t) \equiv & \int_{\Omega}|f(t, x)|^{q+1} d x+\int_{0}^{t} \int_{\Omega}\left|D_{x} f(\tau, x)\right|^{p+1} d x d \tau \\
& +\int_{0}^{t} \int_{\Omega}\left|f_{\tau}^{\prime}(\tau, x)\right|^{\frac{p+1}{p-q+1}} d x d \tau \\
& +\left(\int_{0}^{t}\left(\int_{\Omega}\left|f_{\tau}^{\prime}(\tau, x)\right|^{q+1} d x\right)^{\frac{1}{q+1}} d \tau\right)^{q+1} \quad \forall t<T .
\end{aligned}
$$

ОПРЕДЕЛЕНИЕ 2.1. Будем говорить, что функция $u(t, x)$ является әнергетическим обобщенным решением задачи (2.1)-(2.3), если для любого $T_{0}<T$ :

i) $u-f \in L_{p+1}\left(0, T_{0} ; W_{p+1}^{1}(\Omega, \partial \Omega)\right) \cap L_{\infty}\left(0, T_{0} ; L_{q+1}(\Omega)\right)$;

ii) $\left(|u|^{q-1} u\right)_{t}^{\prime} \in L_{\frac{p+1}{p}}\left(0, T_{0} ;\left(W_{p+1}^{1}(\Omega, \partial \Omega)\right)^{*}\right)$ и выполнено начальное условие (2.3) в смысле

$$
\int_{0}^{T_{0}}\left\langle\left(|u|^{q-1} u\right)_{t}^{\prime}, \zeta\right\rangle d t+\int_{0}^{T_{0}} \int_{\Omega}\left(|u|^{q-1} u-\left|u_{0}\right|^{q-1} u_{0}\right) \zeta_{t}^{\prime} d x d t=0
$$

для произвольной пробной функции $\zeta(t, x) \in L_{p+1}\left(0, T_{0} ; W_{p+1}^{1}(\Omega, \partial \Omega)\right) \cap$ $W_{1}^{1}\left(0, T_{0} ; L_{\infty}(\Omega)\right)$, обращаюшейся в нуль в окрестности $t=T_{0}$; iii) $\begin{aligned} & a_{i}\left(t, x, u(t, x), D_{x} u(t, x)\right) \in L_{\frac{p+1}{p}}\left(\left(0, T_{0}\right) \times \Omega\right), i=1, \ldots, n, \text { и выполняется } \\ & \text { интегральное тождество }\end{aligned}$

$$
\int_{0}^{T_{0}}\left\langle\left(|u|^{q-1} u\right)_{t}^{\prime}, \eta\right\rangle d t+\int_{0}^{T_{0}} \int_{\Omega} \sum_{i=1}^{n} a_{i}\left(t, x, u, D_{x} u\right) \eta_{x_{i}} d x d t=0
$$

где $\eta(t, x)$ - произвольная функция из $L_{p+1}\left(0, T_{0} ; W_{p+1}^{1}(\Omega, \partial \Omega)\right)$.

Через $W_{r}^{1}(\Omega, S)$ обозначаем замькание в норме $W_{r}^{1}(\Omega)$ множества функций из $C^{\infty}(\Omega)$, обращающихся в нуль в окрестности $S \subset \partial \Omega, W_{r}^{1}(\Omega) \equiv W_{r}^{1}(\Omega, \varnothing)$.

Мы не будем рассматривать вопрос о разрешимости задачи (2.1)-(2.3) в общих предположениях. Приведем только известные результаты. Сформулируем сначала дополнительные ограничения на структуру уравнения (2.1):

1) функции $a_{i}, i=1, \ldots, n$, непрерьвны по всем своим аргументам и удовлетворяют следуюшему условию монотонности:

$$
\sum_{i=1}^{n}\left(a_{i}(t, x, s, \xi)-a_{i}(t, x, s, \eta)\right)\left(\xi_{i}-\eta_{i}\right) \geqslant \delta|\xi-\eta|^{p+1}, \quad \delta>0
$$

$2)$ функции $u_{0}(x)$ и $\tilde{f}(t, x)$ удовлетворяют условию согласования:

$$
f(0, x)-u_{0}(x) \in W_{p+1}^{1}(\Omega, \partial \Omega) ;
$$

$3)$ функции $a_{i}, i=1, \ldots, n$, не зависят от $(t, x)$ и

$$
\sum_{i=1}^{n}\left|a_{i}(s, \xi)-a_{i}(v, \xi)\right|^{\frac{p+1}{p}} \leqslant c\left(1+|s|^{q+1}+|v|^{q+1}+|\xi|^{p+1}\right)|s-v| \forall s, v \in \mathbb{R}^{1} .
$$


Введем монотонно возрастающую последовательность $\left\{T_{j}\right\}, T_{j} \rightarrow T$ при $j \rightarrow \infty$, $T_{j}<T$. При выполнении условий 1$\left.), 2\right)$, как следует из [18], при любом $j \in \mathbb{N}$ существует по крайней мере одно энергетическое решение $u_{j}(t, x)$ задачи $(2.1)-(2.3)$ в области $Q_{j}=\left(0, T_{j}\right) \times \Omega$ в смысле определения 2.1. То есть сушествуют функции $u_{j}(t, x)$, удовлетворяющие условиям i), ii), iii) из определения 2.1 с $T_{j}$ вместо $T_{0}$.

Пусть теперь выполнены все дополнительные предположения 1), 2), 3). Тогда, как следует из [19], указанные решения $u_{j}(t, x)$ являются единственными решениями задачи (2.1)-(2.3) в областях $Q_{j}$. Следовательно,

$$
u_{j}(t, x)=u_{i}(t, x) \text { для почти всех }(t, x) \in Q_{i}, \forall j, i \in \mathbb{N}, j>i .
$$

Это значит, что последовательность $\left\{u_{j}\right\}$ определяет единую функцию $u(t, x)$ в области $Q$, являюшуюся, как легко проверить, решением задачи (2.1)-(2.3) в смысле определения 2.1 .

ЗАмЕчАнИЕ 2.1. В силу условия (2.7) решение из определения 2.1 обладает свойством конечности скорости распространения носителя (см., например, [15]), откуда в силу условия (2.4) следует, что при любом граничном режиме (т.е. при любом росте функции $F(t)$ при $t \rightarrow T$ ) существует $T^{\prime}=T^{\prime}(F, R), 0<T^{\prime} \leqslant T$, такое, что

$$
\zeta(t) \equiv \inf \{r: \operatorname{supp} u(t, \cdot) \subset B(r)\}<R \quad \forall t<T^{\prime} .
$$

ОПРЕДЕЛЕНИЕ 2.2. Мы будем говорить, что задача (2.1)-(2.3) обладает свойством локализации, если ее произвольное энергетическое решение $u(t, x)$ имеет следующее свойство:

$$
\zeta(t)<R \quad \forall t<T
$$

ТЕОРЕМА 1. Пусть выполнены структурные условия (2.5)-(2.7) и условие (2.4) на начальную функцию $u_{0}$. Пусть граничный режсим таков, что

$$
F(t) \leqslant \omega(T-t)^{-\alpha_{1}} \quad \forall t<T, \quad \alpha_{1}=\frac{q+1}{p-q}, \quad \omega=\text { const }<\infty .
$$

Тогда существуют постоянние $R_{0}=R_{0}\left(d, d_{0}, d_{1}, q, p,\left\|u_{0}\right\|_{L_{q+1}(\Omega)}\right)<\infty u$ $K=K(R)>0$ такие, что задача (2.1)-(2.4) обладает свойством локализации, как только $R>R_{0}, \omega<K$.

ЗАмЕчАНИЕ 2.2 (о точности условия (2.14)). Задача (1.1)-(1.3), записанная относительно новой неизвестной функции

$$
v(t, x)=u(t, x)^{m}
$$

является частным случаем задачи (2.1)-(2.3) с параметрами

$$
p=1, \quad q=\frac{1}{m}, \quad n=1
$$

и граничным режимом

$$
\tilde{f}(t)=f(t)^{m}
$$


Предельному с точки зрения условий (1.9), (1.11), (1.12) режиму

$$
f(t)=k(T-t)^{-\frac{1}{m-1}}
$$

соответствует

$$
\tilde{f}(t)=k^{m}(T-t)^{-\frac{m}{m-1}}=k^{\frac{1}{q}}(T-t)^{-\frac{1}{1-q}} .
$$

Зафиксируем теперь некоторую функцию $\psi(x) \in C^{1}[0, \infty), \psi(0)=1, \operatorname{supp} \psi \in$ $[0, R)$, и в качестве продолжения $f(t, x)$ возьмем

$$
f(t, x) \equiv \tilde{f}(t) \psi(x)
$$

Тривиальные вычисления показывают, что для функции граничного режима $F(t)$, построенной по функции $f(t, x)$ по формуле (2.11), справедлива оценка:

$$
F(t) \leqslant c(T-t)^{-\frac{q+1}{1-q}} \quad \forall t<T, \quad c=\text { const }<\infty,
$$

и, следовательно, теорема 1 гарантирует наличие локализации.

ОПРЕДЕЛЕНИЕ 2.3. Измеримое множество $G \subset \bar{\Omega}$ будем называть множеством регулярности решения $u(t, x)$ задачи $(2.1)-(2.3)$, если

$$
\int_{G}|u(t, x)|^{q+1} d x+\int_{(0, t) \times G}|u(\tau, x)|^{p+1} d x d \tau<c=c(G)<\infty \quad \forall t<T .
$$

ОПРЕДЕЛЕНИЕ 2.4. Множество $S \subset \bar{\Omega}$ называется множеством сингулярности некоторого решения $u(t, x)$ задачи (2.1)-(2.3), если

$$
S=\bar{\Omega} \backslash(\bigcup G)
$$

где справа стоит объединение всех множеств регулярности.

ТЕОРема 2. Пусть в условиях теоремь 1 функиия граничного режимм $F(t)$ удовлетворяет дополнительному ограничению

$$
F(t) \leqslant \omega(t)(T-t)^{-\alpha_{1}} \quad \forall t<T, \quad \alpha_{1}=\frac{q+1}{p-q},
$$

где $\omega(t)$ - произвольная монотонно невозрастающая непрерывная неотрицательная функиия, удовлетворяющая лищь одному условию:

$$
\omega(t) \rightarrow 0 \quad \text { npu } \quad t \rightarrow T
$$

Тогда для произвольного обобщенного решения задачи (2.1)-(2.3) мнохсество сингулярности

$$
S \subset \partial B(1)
$$

т.е. $\bar{\Omega} \backslash B(r)$ является множеством регулярности $\forall r>1$.

ЗАмЕчАНИЕ 2.3. Модельность области $\Omega$ в задаче (2.1)-(2.3) определялась лишь желанием избежать дополнительных громоздких, но достаточно очевидных построений и вычислений. Все результаты остаются справедливыми для областей вида $B(R) \cap\left(\mathbb{R}^{n} \backslash \Omega\right), R<\infty$. Здесь $\Omega$ - произвольная односвязная область с $C^{1}$-гладкой гранищей $\partial \Omega: \bar{\Omega} \subset B(R)$. 


\section{§ 3. Предварительные сведения и вспомогательные построения и утверждения}

Пусть $G$-произвольная ограниченная область в $\mathbb{R}^{n}$ с $C^{1}$-гладкой границей $\partial G$, пусть $G_{r}$ - область, гомотетичная области $G$ с коэффициентом гомотетии $r>0$. Тогда хорошо известно следуюшее интерполяционное неравенство (см. [15]):

$$
\|v\|_{L_{p+1}\left(\partial G_{r}\right)} \leqslant C\left(\left\|D_{x} v\right\|_{L_{p+1}\left(G_{r}\right)}+r^{-\delta}\|v\|_{L_{q+1}\left(G_{r}\right)}\right)^{\theta}\|v\|_{L_{q+1}\left(G_{r}\right)}^{1-\theta},
$$

где $v(x)$ - произвольная функция из соболевского пространства $W_{p+1}^{1}\left(G_{r}\right), 0 \leqslant q \leqslant$ $p<\infty$,

$$
\delta=\frac{n(p-q)+(p+1)(q+1)}{(p+1)(q+1)}, \quad \theta=\frac{n(p-q)+q+1}{n(p-q)+(q+1)(p+1)}
$$

универсальная постоянная $C<\infty$ зависит только от известных параметров и не зависит, в частности, от $v(x), r$.

Введем теперь следующие семейства подобластей, связанных с областями $\Omega, Q$ задачи (2.1)-(2.3):

$$
\begin{gathered}
\Omega(s)=\Omega_{R} \backslash B(s) \quad \forall s>1 \\
Q_{t_{1}}^{t_{2}}(s)=\left(t_{1}, t_{2}\right) \times \Omega(s), \quad 0 \leqslant t_{1}<t_{2}<T .
\end{gathered}
$$

Исходя из неравенства (3.1) легко выводится следующее интерполяционное неравенство для введенного семейства ограниченных областей $\Omega(s)$ :

$$
\|v\|_{L_{p+1}(\partial \Omega(s))}<C_{1}\left\|D_{x} v\right\|_{L_{p+1}(\Omega(s))}^{\theta}\|v\|_{L_{q+1}(\Omega(s))}^{1-\theta} \quad \forall s>1
$$

где $v(x)$ - произвольная функция из $W_{p+1}^{1}(\Omega(s), \partial B(R)), \theta-$ из $(3.1)$, постоянная $C_{1}<\infty$ не зависит от $v(x), s>1$.

Лемма 3.1. Для почти всех $a, b, 0 \leqslant a<b<T$, имеет место следующая глобальная априорная оченка произвольного энергетического решения $u(t, x)$ задачи (2.1)-(2.3):

$$
\begin{aligned}
\int_{\Omega}|u(b, x)|^{q+1} d x & +\int_{a}^{b} \int_{\Omega}\left|D_{x} u\right|^{p+1} d x d t \\
& \leqslant c_{1} \int_{\Omega}|u(a, x)|^{q+1} d x+c_{2}\left(1+\zeta_{1}(b)^{\frac{q(p+1)}{p-q+1}}\right) F_{1}(a, b),
\end{aligned}
$$

әде $\zeta_{1}(s)=\sup _{0 \leqslant \tau<s} \zeta(\tau), \zeta(\tau)-$ из определения 2.2 ;

$$
\begin{aligned}
F_{1}(a, b) \equiv \int_{\Omega} \mid & \left.f(b, x)\right|^{q+1} d x+\int_{a}^{b} \int_{\Omega}\left|D_{x} f\right|^{p+1} d x d t \\
& +\left(\int_{a}^{b}\left\|f_{t}^{\prime}\right\|_{L_{q+1}(\Omega)} d t\right)^{q+1}+\int_{a}^{b}\left\|f_{t}^{\prime}\right\|_{L_{p_{1}}(\Omega)}^{p_{1}} d t ; \quad F_{1}(0, b) \equiv F(b) ;
\end{aligned}
$$

здесь $p_{1}=\frac{p+1}{p-q+1}$. 
ДокАЗАТЕЛЬСТво. В силу формулы интегрирования по частям, установленной в [18], для почти всех $0 \leqslant a<b<T$ имеем равенство:

$$
\begin{aligned}
\int_{a}^{b}\left\langle\left(|u|^{q-1} u\right)_{t}^{\prime}, u-f\right\rangle d t & \\
= & \frac{1}{q+1} \int_{\Omega}\left(|u(b, x)|^{q+1}-|u(a, x)|^{q+1}\right) d x \\
& \quad+\int_{a}^{b} \int_{\Omega}\left(|u(t, x)|^{q-1} u(t, x)-|u(a, x)|^{q-1} u(a, x)\right) f_{t}^{\prime}(t, x) d x d t \\
& -\int_{\Omega}\left(|u(b, x)|^{q-1} u(b, x)-|u(a, x)|^{q-1} u(a, x)\right) f(b, x) d x
\end{aligned}
$$

Поэтому, подставляя в интегральное тождество (2.12) в качестве пробной функции $u(t, x)-f(t, x)$, получаем с учетом равенства (3.4) и структурных условий $(2.5),(2.6)$ :

$$
\begin{aligned}
\frac{q}{q+1} & \int_{\Omega}|u(b, x)|^{q+1} d x+d_{0} \int_{a}^{b} \int_{\Omega}\left|D_{x} u\right|^{p+1} d x d t \\
\leqslant & \int_{a}^{b} \int_{\Omega}\left|D_{x} u\right|^{p}\left|D_{x} f\right| d x d t+\int_{\Omega}\left(|u(b, x)|^{q}+|u(a, x)|^{q}\right)|f(b, x)| d x \\
& -\int_{a}^{b} \int_{\Omega}\left(|u(t, x)|^{q-1} u(t, x)-|u(a, x)|^{q-1} u(a, x)\right) f_{t}^{\prime}(t, x) d x d t \\
& +\frac{q}{q+1} \int_{\Omega}|u(a, x)|^{q+1} d x
\end{aligned}
$$

Оценим сверху стандартньм образом слагаемые справа в неравенстве (3.5):

$$
\begin{gathered}
\int_{a}^{b} \int_{\Omega}\left|D_{x} u\right|^{p}\left|D_{x} f\right| d x d t \\
\leqslant \varepsilon_{1} \int_{a}^{b} \int_{\Omega}\left|D_{x} u\right|^{p+1} d x d t+c_{1}\left(\varepsilon_{1}\right) \int_{a}^{b} \int_{\Omega}\left|D_{x} f\right|^{p+1} d x d t \\
\int_{\Omega}\left(|u(b, x)|^{q}+|u(a, x)|^{q}\right)|f(b, x)| d x \\
\leqslant \varepsilon_{1}\left[\|u(b, x)\|_{L_{q+1}(\Omega)}^{q+1}+\|u(a, x)\|_{L_{q+1}(\Omega)}^{q+1}\right] \\
+c_{2}\left(\varepsilon_{1}\right)\|f(b, x)\|_{L_{q+1}(\Omega)}^{q+1} \quad \forall \varepsilon_{1}>0
\end{gathered}
$$




$$
\begin{gathered}
\int_{a}^{b} \int_{\Omega}|u(a, x)|^{q}\left|f_{t}^{\prime}(t, x)\right| d x d t \\
\leqslant \int_{a}^{b}\left(\int_{\Omega}|u(a, x)|^{q+1} d x\right)^{\frac{q}{q+1}}\left(\int_{\Omega}\left|f_{t}^{\prime}\right|^{q+1} d x\right)^{\frac{1}{q+1}} d t \\
=\|u(a, x)\|_{L_{q+1}(\Omega)}^{q} \cdot \int_{a}^{b}\left(\int_{\Omega}\left|f_{t}^{\prime}(t, x)\right|^{q+1} d x\right)^{\frac{1}{q+1}} d t \\
\leqslant \varepsilon_{1}\|u(a, x)\|_{L_{q+1}(\Omega)}^{q+1} \\
\quad+c_{3}\left(\varepsilon_{1}\right)\left(\int_{a}^{b}\left(\int_{\Omega}\left|f_{t}^{\prime}\right|^{q+1} d x\right)^{\frac{1}{q+1}} d t\right)^{q+1} \forall \varepsilon_{1}>0 ; \\
\int_{a}^{b} \int_{\Omega}|u(t, x)|^{q}\left|f_{t}^{\prime}(t, x)\right| d x d t \\
\leqslant \int_{a}^{b}\left(\int_{\Omega}|u(t, x)|^{p+1} d x\right)^{\frac{q}{p+1}}\left(\int_{\Omega}\left|f_{t}^{\prime}(t, x)\right|^{\frac{p+1}{p-q+1}} d x\right)^{\frac{p-q+1}{p+1}} d t .
\end{gathered}
$$

В силу неравенства Пуанкаре имеем:

$$
\int_{\Omega}|u(t, x)|^{p+1} d x \leqslant \zeta(t)^{p+1} \int_{\Omega}\left|D_{x} u(t, x)\right|^{p+1} d x,
$$

поэтому, продолжая предпоследнее неравенство, получим:

$$
\begin{aligned}
& \int_{a}^{b} \int_{\Omega}|u(t, x)|^{q}\left|f_{t}^{\prime}(t, x)\right| d x d t \\
& \quad \leqslant \int_{a}^{b} \zeta(t)^{q}\left(\int_{\Omega}\left|D_{x} u\right|^{p+1} d x\right)^{\frac{q}{p+1}}\left(\int_{\Omega}\left|f_{t}^{\prime}\right|^{\frac{p+1}{p-q+1}} d x\right)^{\frac{p-q+1}{p+1}} d t \\
& \quad \leqslant \varepsilon_{2} \int_{a}^{b} \int_{\Omega}\left|D_{x} u\right|^{p+1} d x d t+c_{4}\left(\varepsilon_{2}\right) \zeta_{1}(b)^{\frac{q(p+1)}{p+q+1}} \int_{a}^{b} \int_{\Omega}\left|f_{t}^{\prime}\right|^{\frac{p+1}{p-q+1}} d x d t \quad \forall \varepsilon_{2}>0 .
\end{aligned}
$$

Объединяя полученные оценки, выводим из неравенства (3.5):

$$
\begin{aligned}
& \left(\frac{q}{q+1}-\varepsilon_{1}\right) \int_{\Omega}|u(b, x)|^{q+1} d x+\left(d_{0}-\varepsilon_{1}-\varepsilon_{2}\right) \int_{a}^{b} \int_{\Omega}\left|D_{x} u\right|^{p+1} d x d t \\
& \leqslant\left(\frac{q}{1+q}+2 \varepsilon_{1}\right) \int_{\Omega}|u(a, x)|^{q+1} d x+c_{2}\left(\varepsilon_{1}\right)\|f(b, x)\|_{L_{q+1}(\Omega)}^{q+1} \\
& \quad+c_{1}\left(\varepsilon_{1}\right) \int_{a}^{b} \int_{\Omega}\left|D_{x} f\right|^{p+1} d x d t+c_{3}\left(\varepsilon_{1}\right)\left(\int_{a}^{b}\left\|f_{t}^{\prime}(t, x)\right\|_{L_{q+1}(\Omega)} d t\right)^{q+1} \\
& \quad+c_{4}\left(\varepsilon_{2}\right) \zeta_{1}(b)^{\frac{q(p+1)}{p-q+1}} \int_{a}^{b}\left\|f_{t}^{\prime}(t, x)\right\|_{L_{p_{1}}(\Omega)}^{p_{1}} d t, \quad p_{1}=\frac{p+1}{p-q+1} .
\end{aligned}
$$

Фиксируя теперь $\varepsilon_{1}>0, \varepsilon_{2}>0$ так, что

$$
\frac{q}{q+1}-\varepsilon_{1} \geqslant \frac{q}{2(q+1)}, \quad\left(d_{0}-\varepsilon_{1}-\varepsilon_{2}\right) \geqslant \frac{d_{0}}{2},
$$

приходим к необходимой оценке (3.3). 
ЛЕмма 3.2. Пусть $и(t, x)$ - произвольное обобщенное решение задачи (2.1)-(2.3). Тогда в условиях леммы 3.1 для почти всех $s>1$ выполняется следующее соотношение:

$$
\begin{gathered}
\int_{\Omega(s)}|u(b, x)|^{q+1} d x+\frac{d_{0}(q+1)}{q} \int_{Q_{a}^{b}(s)}\left|D_{x} u\right|^{p+1} d x d t \\
\leqslant \int_{\Omega(s)}|u(a, x)|^{q+1} d x+c\left(\int_{\Gamma_{a}^{b}(s)}\left|D_{x} u\right|^{p+1} d \gamma d t\right)^{\frac{p}{p+1}}\left(\int_{Q_{a}^{b}(s)}\left|D_{x} u\right|^{p+1} d x d t\right)^{\frac{\theta}{p+1}} \\
\times\left(\int_{a}^{b}\left(\int_{\Omega(s)}|u|^{q+1} d x\right)^{\frac{p+1}{q+1}} d t\right)^{\frac{1-\theta}{p+1}}
\end{gathered}
$$

где $\theta-$-из (3.1), $с<\infty$ зависит только от известных параметров задачи $(2.1)-(2.3), \Gamma_{a}^{b}(s)=(a, b) \times \partial B(s)$.

ДокАЗАТЕЛьство. Зафиксируем $s>1, \delta>0$ и введем липшицеву срезающую функцию $\eta_{s, \delta}(\tau): \eta_{s, \delta}(\tau)=0$ при $\tau<s, \eta_{s, \delta}(\tau)=1$ при $\tau>s+\delta, \eta_{s, \delta}(\tau)=(\tau-s) / \delta$ при $s \leqslant \tau \leqslant s+\delta$. Подставим в интегральное тождество (2.12) в качестве пробной функции $\eta(t, x) \equiv u(t, x) \eta_{s, \delta}(|x|)$, запишем сначала равенство с $T_{0}=b$, затем с $T_{0}=a$ и вычтем второе из первого. Используя формулу интегрирования по частям из [18], получаем

$$
\begin{aligned}
\frac{q}{q+1} & \int_{\Omega(s)}|u(b, x)|^{q+1} \eta_{s, \delta}(|x|) d x+\int_{Q_{a}^{b}(s)} \sum_{i=1}^{n} a_{i}\left(\ldots, D_{x} u\right) u_{x_{i}} \cdot \eta_{s, \delta}(|x|) d x d t \\
= & \frac{q}{q+1} \int_{\Omega(s)}|u(a, x)|^{q+1} \eta_{s, \delta}(|x|) d x \\
& -\int_{Q_{a}^{b}(s) \backslash Q_{a}^{b}(s+\delta)} \sum_{i=1}^{n} a_{i}\left(\ldots, D_{x} u\right) u \cdot\left(\eta_{s, \delta}(|x|)\right)_{x_{i}} d x d t .
\end{aligned}
$$

Отсюда, переходя к пределу при $\delta \rightarrow 0$ (см. [15]), приходим в силу условий (2.5), (2.6) к неравенству

$$
\begin{aligned}
\frac{q}{q+1} \int_{\Omega(s)}|u(b, x)|^{q+1} d x+\int_{Q_{a}^{b}(s)} \sum_{i=1}^{n} a_{i}\left(\ldots, D_{x} u\right) u_{x_{i}} d x d t \\
=\frac{q}{q+1} \int_{\Omega(s)}|u(a, x)|^{q+1} d x+\int_{\Gamma_{a}^{b}(s)} \sum_{i=1}^{n} a_{i}\left(\ldots, D_{x} u\right) u \nu_{i} d \gamma d t \\
\leqslant \frac{q}{q+1} \int_{\Omega(s)}|u(a, x)|^{q+1} d x \\
\quad+c\left(\int_{\Gamma_{a}^{b}(s)}\left|D_{x} u\right|^{p+1} d \gamma d t\right)^{\frac{p}{p+1}}\left(\int_{\Gamma_{a}^{b}(s)}|u|^{p+1} d \gamma d t\right)^{\frac{1}{p+1}},
\end{aligned}
$$

где $\boldsymbol{\nu}=\left(\nu_{1}, \ldots, \nu_{n}\right)$ - вектор единичной нормали к $\partial B(s)$. 
Оценивая $\int_{\partial B(s)}|u(t, x)|^{p+1} d \gamma$ при помощи интерполяционного неравенства (3.2) и интегрируя по $t$ в интервале $(a, b)$, непосредственно из последнего неравенства выводим доказываемое соотношение (3.6).

Введем теперь монотонную последовательность

$$
t_{j} \rightarrow T, \quad t_{0}=0, \quad t_{j+1}>t_{j}, j=1,2, \ldots ; \quad \Delta_{j} \equiv t_{j}-t_{j-1},
$$

и определим два семейства функций:

$$
\begin{aligned}
& h_{j}(s)=\operatorname{essiup}_{t \in\left[t_{j-1}, t_{j}\right]} \int_{\Omega(s)}|u(t, x)|^{q+1} d x \quad \forall s>1, \quad j=1,2, \ldots, \\
& E_{j}(s)=\int_{t_{j-1}}^{t_{j}} \int_{\Omega(s)}\left|D_{x} u(t, x)\right|^{p+1} d x d t \quad \forall s>1, \quad j=1,2, \ldots,
\end{aligned}
$$

где $u(t, x)$ - рассматриваемое обобшенное решение задачи (2.1)-(2.3).

ЛЕМма 3.3. Пусть выполнены все предположсения теоремы 1 и $и(t, x)$ произвольное энергетическое решение рассматриваемой задачи. Тогда для введенных выше семейств функиий $\left\{h_{j}(s)\right\},\left\{E_{j}(s)\right\}$, связанных с $u(t, x)$, выьполняется следующая система дифференциальных неравенств:

$$
\begin{gathered}
E_{j}(s) \leqslant r_{1} h_{j-1}(s)+r_{2} \Delta_{j}^{\nu}\left(-\frac{d E_{j}(s)}{d s}\right)^{1+\mu} \quad \forall s>1, \quad j \in \mathbb{N}, \\
h_{j}(s) \leqslant\left(1+\delta_{j}\right) h_{j-1}(s)+r_{3} \delta_{j}^{-\frac{(p+1) \nu}{q+1}} \Delta_{j}^{\nu}\left(-\frac{d E_{j}(s)}{d s}\right)^{1+\mu} \\
\forall s>1, \quad j \in \mathbb{N}, \quad \forall \delta_{j}>0,
\end{gathered}
$$

әде постоянные $r_{1}, r_{2}, r_{3}<\infty$ зависят лишь от известных параметров,

$$
\nu=\frac{(1-\theta)(q+1)}{q(p+1)+\theta(p-q)}<1, \quad \mu=\frac{(1-\theta)(p-q)}{q(p+1)+\theta(p-q)}, \quad \theta-u з \text { (3.1). }
$$

ДокАЗАТЕЛЬСТво. Используя легко проверяемое соотношение

$$
\int_{t_{j-1}}^{t_{j}} \int_{\partial B(s)}\left|D_{x} u\right|^{p+1} d \gamma d t=-\frac{d}{d s} E_{j}(s)
$$

вьводим из неравенства (3.6) при $a=t_{j-1}, b=t_{j}$ :

$$
\begin{aligned}
E_{j}(s) \leqslant & \frac{q}{d_{0}(q+1)} \int_{\Omega(s)}\left|u\left(t_{j-1}, x\right)\right|^{q+1} d x \\
& +c_{1} E_{j}(s)^{\frac{\theta}{p+1}}\left(-E_{j}^{\prime}(s)\right)^{\frac{p}{p+1}}\left(\int_{t_{j-1}}^{t_{j}}\left(\int_{\Omega(s)}|u|^{q+1} d x\right)^{\frac{p+1}{q+1}} d t\right)^{\frac{1-\theta}{p+1}} \\
\leqslant & \frac{q}{d_{0}(q+1)} h_{j-1}(s)+c_{1} E_{j}(s)^{\frac{\theta}{p+1}}\left(-E_{j}^{\prime}(s)\right)^{\frac{p}{p+1}} h_{j}(s)^{\frac{1-\theta}{q+1}} \Delta_{j}^{\frac{1-\theta}{p+1}}
\end{aligned}
$$


Снова возврашаясь к неравенству (3.6), полагая в нем $a=t_{j-1}, b=\tilde{t} \in\left(t_{j-1}, t_{j}\right]$, получим, что для почти всех $\tilde{t}$ из указанного интервала имеет место оценка:

$$
\begin{aligned}
\int_{\Omega(s)}|u(\tilde{t}, x)|^{q+1} d x & \leqslant \int_{\Omega(s)}\left|u\left(t_{j-1}, x\right)\right|^{q+1} d x \\
& +c_{2}\left(\int_{t_{j-1}}^{\tilde{t}} \int_{\partial B(s)}\left|D_{x} u\right|^{p+1} d \gamma d t\right)^{\frac{p}{p+1}} \\
\times & \left(\int_{t_{j-1}}^{\tilde{t}} \int_{\Omega(s)}\left|D_{x} u\right|^{p+1} d x d t\right)^{\frac{\theta}{p+1}}\left(\int_{t_{j-1}}^{\tilde{t}}\left(\int_{\Omega(s)}|u|^{q+1} d x\right)^{\frac{p+1}{q+1}} d t\right)^{\frac{1-\theta}{p+1}} .
\end{aligned}
$$

Отсюда ввиду произвольности $\tilde{t} \in\left(t_{j-1}, t_{j}\right]$ легко выводим:

$$
h_{j}(s) \leqslant h_{j-1}(s)+c_{3} h_{j}(s)^{\frac{1-\theta}{q+1}} \Delta_{j}^{\frac{1-\theta}{p+1}} E_{j}(s)^{\frac{\theta}{p+1}}\left(-E_{j}^{\prime}(s)\right)^{\frac{p}{p+1}} .
$$

Складывая теперь неравенства (3.10), (3.11), а затем применяя неравенство Юнга с " $\varepsilon$ ", получаем:

$$
\begin{aligned}
h_{j}(s) & +E_{j}(s) \\
\leqslant & \left(1+\frac{q}{d_{0}(q+1)}\right) h_{j-1}(s)+c_{3} h_{j}(s)^{\frac{1-\theta}{q+1}} \Delta_{j}^{\frac{1-\theta}{p+1}} E_{j}(s)^{\frac{\theta}{p+1}}\left(-E_{j}^{\prime}(s)\right)^{\frac{p}{p+1}} \\
\leqslant & \left(1+\frac{q}{d_{0}(q+1)}\right) h_{j-1}(s)+\varepsilon h_{j}(s)+\varepsilon E_{j}(s)+c_{4}(\varepsilon) \Delta_{j}^{\nu}\left(-E_{j}^{\prime}(s)\right)^{1+\mu},
\end{aligned}
$$

где $\nu$ и $\mu$-из (3.8), (3.9). Полагая в последнем неравенстве $\varepsilon=1 / 2$, приходим к доказываемому соотношению $(3.8) \mathrm{c} r_{1}=2\left(1+\frac{q}{d_{0}(q+1)}\right), r_{2}=2 c_{4}(1 / 2)$.

Теперь об оценке (3.9). Для любого сколь угодно малого $\varepsilon>0$ выберем такое $t^{\prime} \in\left(t_{j-1}, t_{j}\right], t^{\prime}=t^{\prime}(\varepsilon)$, что

$$
(1-\varepsilon) h_{j}(s)=\int_{\Omega(s)}\left|u\left(t^{\prime}, x\right)\right|^{q+1} d x .
$$

Теперь из соотношения (3.6) при $a=t_{j-1}, b=t^{\prime}$ получаем:

$$
\begin{aligned}
(1-\varepsilon) & h_{j}(s)+\frac{d_{0}(q+1)}{q} \int_{t_{j-1}}^{t^{\prime}} \int_{\Omega(s)}\left|D_{x} u\right|^{p+1} d x d t \\
\leqslant & h_{j-1}(s)+c h_{j}(s)^{\frac{1-\theta}{1+q}} \Delta_{j}^{\frac{1-\theta}{p+1}}\left(\int_{t_{j-1}}^{t^{\prime}} \int_{\Omega(s)}\left|D_{x} u\right|^{p+1} d x d t\right)^{\frac{\theta}{p+1}} \\
& \times\left(\int_{t_{j-1}}^{t^{\prime}} \int_{\partial B(s)}\left|D_{x} u\right|^{p+1} d \gamma d t\right)^{\frac{p}{p+1}} \\
= & h_{j-1}(s)+\left(\varepsilon h_{j}(s)\right)^{\frac{1-\theta}{1+q}}\left(h \int_{t_{j-1}}^{t_{j}} \int_{\Omega(s)}\left|D_{x} u\right|^{p+1} d x d t\right)^{\frac{\theta}{p+1}} \\
& \times\left(c^{\frac{p+1}{p}} h^{-\frac{\theta}{p}} \int_{t_{j-1}}^{t^{\prime}} \int_{\partial B(s)}\left|D_{x} u\right|^{p+1} d \gamma d t\right)^{\frac{p}{p+1}} \\
& \times\left(\varepsilon^{-\frac{p+1}{q+1}} \Delta_{j}\right)^{\frac{1-\theta}{p+1}} \quad \forall \varepsilon>0, \quad \forall h>0 .
\end{aligned}
$$


Отсюда в силу неравенства Юнга получаем:

$$
\begin{gathered}
(1-2 \varepsilon) h_{j}(s)+\left[\frac{d_{0}(q+1)}{q}-h\right] \int_{t_{j-1}}^{t^{\prime}} \int_{\Omega(s)}\left|D_{x} u\right|^{p+1} d x d t \\
\leqslant h_{j-1}(s)+\left(\varepsilon^{-\frac{p+1}{q+1}} \Delta_{j}\right)^{\nu}\left(c^{\frac{p+1}{p}} h^{-\frac{\theta}{p}} \int_{t_{j-1}}^{t^{\prime}} \int_{\partial B(s)}\left|D_{x} u\right|^{p+1} d \gamma d t\right)^{1+\mu} \\
\forall \varepsilon>0, \quad \forall h>0 .
\end{gathered}
$$

Фиксируя в этом соотношении $h=\frac{d_{0}(q+1)}{2 q}$ и полагая $\varepsilon=\frac{\delta_{j}}{2\left(1+\delta_{j}\right)}$, приходим к требуемой оценке (3.9).

\section{§4. Достаточное условие локализации решения}

В настояшем параграфе проводится доказательство теоремы 1 на основе изучения свойств функций, удовлетворяющих бесконечной дифференциальной системе $(3.8),(3.9)$. Сначала детализируем выбор последовательности $\left\{t_{j}\right\}$. Зафиксируем два числа

$$
0<\xi_{1}<\xi_{2}<1
$$

и выбор точек последовательности $\left\{t_{j}\right\}$ обусловим лишш одним ограничением:

$$
\xi_{1}<\frac{\Delta_{i+1}}{\Delta_{i}}<\xi_{2} \quad \forall i \in \mathbb{N}
$$

При этом, как несложно убедиться, будут выполняться соотношения:

$$
\frac{\xi_{1}}{1-\xi_{1}} \Delta_{j} \leqslant T-t_{j} \equiv \sum_{i=j+1}^{\infty} \Delta_{i} \leqslant \frac{\xi_{2}}{1-\xi_{2}} \Delta_{j} \quad \forall j \in \mathbb{N}
$$

Теперь введем нормированные функции:

$$
A_{j}(s)=\Delta_{j}^{\frac{q+1}{p-q}} E_{j}(s), \quad H_{j}(s) \equiv \Delta_{j}^{\frac{q+1}{p-q}} h_{j}(s), \quad j=1,2, \ldots
$$

При этом соотношения (3.8), (3.9) эквивалентны соотношениям:

$$
\begin{aligned}
& A_{j}(s) \leqslant r_{4} H_{j-1}(s)+r_{2}\left(-A_{j}^{\prime}(s)\right)^{1+\mu} \quad \forall s>1, \quad r_{4}=r_{1} \xi_{2}^{\frac{q+1}{p-q}} \\
& H_{j}(s) \leqslant\left(1+\delta_{j}\right) \xi_{2}{ }^{\frac{q+1}{p-q}} H_{j-1}(s)+r_{3}^{\prime} \delta_{j}^{-\frac{(p+1) \nu}{q+1}}\left(-A_{j}^{\prime}(s)\right)^{1+\mu} \quad \forall s>1, j \in \mathbb{N},
\end{aligned}
$$

где $H_{0}(s) \equiv T^{\frac{q+1}{p-q}} h_{0}(s)$.

Зафиксируем теперь некоторое $\lambda, 1>\lambda>\xi_{2}^{\frac{q+1}{p-q}}$, и выберем параметры $\delta_{j}$ так, что

$$
\left(1+\delta_{j}\right) \xi_{2}^{\frac{q+1}{p-q}}=\lambda \Longrightarrow \delta_{j}=\delta_{0} \equiv \lambda \xi_{2}^{-\frac{q+1}{p-q}}-1
$$


При этом соотношение (4.4) примет вид:

$$
H_{j}(s) \leqslant \lambda H_{j-1}(s)+r_{3}\left(-A_{j}^{\prime}(s)\right)^{1+\mu}, \quad r_{3}=r_{3}^{\prime}\left(\delta_{0}\right) \delta_{0}^{-\frac{\nu(p+1)}{q+1}} .
$$

Начнем теперь итерировать соотношение (4.3), оценивая при этом все $H_{i}(s)$ при помощи соотношения $\left(4.4^{*}\right)$, и получим:

$$
\begin{aligned}
& A_{j}(s) \leqslant r_{4} H_{j-1}(s)+r_{2}\left(-A_{j}^{\prime}(s)\right)^{1+\mu} \\
& \leqslant r_{4} \lambda H_{j-2}(s)+r_{4} r_{3}\left(-A_{j-1}^{\prime}(s)\right)^{1+\mu}+r_{2}\left(-A_{j}^{\prime}(s)\right)^{1+\mu} \\
& \leqslant r_{4} \lambda^{2} H_{j-3}(s)+r_{4} r_{3} \lambda\left(-A_{j-2}^{\prime}(s)\right)^{1+\mu} \\
& +r_{4} r_{3}\left(-A_{j-1}^{\prime}(s)\right)^{1+\mu}+r_{2}\left(-A_{j}^{\prime}(s)\right)^{1+\mu} \\
& \leqslant \cdots \leqslant r_{4} \lambda^{j-1} H_{0}(s)+r_{4} r_{3}\left[\lambda^{j-2}\left(-A_{1}^{\prime}(s)\right)^{1+\mu}+\lambda^{j-3}\left(-A_{2}^{\prime}(s)\right)^{1+\mu}\right. \\
& +\cdots+\lambda^{2}\left(-A_{j-3}^{\prime}(s)\right)^{1+\mu}+\lambda\left(-A_{j-2}^{\prime}(s)\right)^{1+\mu} \\
& \left.+\left(-A_{j-1}^{\prime}(s)\right)^{1+\mu}+\frac{r_{2}}{r_{3} r_{4}}\left(-A_{j}^{\prime}(s)\right)^{1+\mu}\right] \\
& \leqslant r_{4} \lambda^{j-1} H_{0}(s)+r_{5} \sum_{i=1}^{j}\left(-\lambda^{\frac{j-i}{1+\mu}} A_{j}^{\prime}(s)\right)^{1+\mu}, \\
& \text { где } r_{5}=\frac{r_{3} r_{4}}{\lambda} \max \left(1, \frac{\lambda r_{2}}{r_{3} r_{4}}\right) \text {. }
\end{aligned}
$$

Из неравенства (4.5) в силу выпуклости вниз функции $f(s)=s^{1+\mu}, \mu>0$, вытекает:

$$
A_{j}(s) \leqslant r_{4} \lambda^{j-1} T^{\frac{q+1}{p-q}} h_{0}(s)+r_{5}\left[\sum_{i=1}^{j}\left(-\lambda^{\frac{j-1}{1+\mu}} A_{i}^{\prime}(s)\right)\right]^{1+\mu}
$$

Введем теперь еще одно семейство неотрицательных функций:

$$
U_{j}(s) \equiv \sum_{i=1}^{j} \lambda^{\frac{j-i}{1+\mu}} A_{i}(s), \quad j=1,2, \ldots
$$

Очевидно равенство: $U_{j}(s)-\lambda^{\frac{1}{1+\mu}} U_{j-1}(s)=A_{j}(s), U_{0}(s)=0$; поэтому соотношение (4.6) может быть переписано в виде:

$$
U_{j}(s) \leqslant \lambda^{\frac{1}{1+\mu}} U_{j-1}(s)+r_{4} \lambda^{j-1} T^{\frac{q+1}{p-q}} h_{0}(s)+r_{5}\left(-U_{j}^{\prime}(s)\right)^{1+\mu} \quad \forall j \in \mathbb{N} .
$$

Оценим сверху начальное значение $U_{j}(1)$ :

$$
U_{j}(1)=\sum_{i=1}^{j} \lambda^{\frac{j-i}{1+\mu}} A_{i}(1)=\sum_{i=1}^{j} \lambda^{\frac{j-i}{1+\mu}} \Delta_{i}^{\frac{q+1}{p-q}} E_{i}(1) \quad \forall j \in \mathbb{N} .
$$


Для оценки сверху $E_{j}(1)$ воспользуемся неравенством (3.3) из леммы 3.1. В силу (4.2) имеем:

$$
F_{1}\left(1, t_{j}\right) \equiv F\left(t_{j}\right) \leqslant \omega\left(T-t_{j}\right)^{-\alpha_{1}} \leqslant \omega\left(\frac{1-\xi_{1}}{\xi_{1}}\right)^{\alpha_{1}} \Delta_{j}^{-\frac{q+1}{p-q}}
$$

поэтому из соотношения (3.3) при $a=0, b=t_{j}$ вытекает

$$
E_{j}(1) \leqslant c_{1} h_{0}(1)+c_{3} \omega\left(1+\zeta_{1}\left(t_{j}\right)^{\frac{q(p+1)}{p-q+1}}\right) \Delta_{j}^{-\frac{q+1}{p-q}}, \quad c_{3}=c_{2}\left(\frac{1-\xi_{1}}{\xi_{1}}\right)^{\frac{q+1}{p-q}}
$$

Следовательно,

$$
U_{j}(1) \leqslant c_{1} g_{j} T^{\frac{q+1}{p-q}} h_{0}(1)+c_{3} \omega\left(1-\lambda^{\frac{1}{1+\mu}}\right)^{-1}\left(1+\zeta_{1}\left(t_{j}\right)^{\frac{q(p+1)}{p-q+1}}\right),
$$

где

$$
\begin{array}{ll}
g_{j} \equiv \sum_{i=1}^{j} \lambda^{\frac{j-i}{1+\mu}} 2^{-\frac{i(q+1)}{p-q}} \leqslant \lambda^{\frac{j}{1+\mu}}\left(\lambda^{\frac{1}{1+\mu}} 2^{\frac{q+1}{p-q}}-1\right)^{-1}, & \text { если } \lambda^{\frac{1}{1+\mu}} 2^{\frac{q+1}{p-q}}>1, \\
g_{j} \leqslant j 2^{-\frac{j(q+1)}{p-q}}, & \text { если } \lambda^{\frac{1}{1+\mu}} 2^{\frac{q+1}{p-q}}=1, \\
g_{j} \leqslant 2^{-\frac{j(q+1)}{p-q}}\left(1-\lambda^{\frac{1}{1+\mu}} 2^{\frac{q+1}{p-q}}\right), & \text { если } \lambda^{\frac{1}{1+\mu}} 2^{\frac{1+q}{p-q}}<1 .
\end{array}
$$

Очевидно, что $g_{j} \leqslant g_{0}=$ const $<\infty$, где $g_{0}$ не зависит от $j \in \mathbb{N}$. Следовательно, полученная оценка для $U_{j}(1)$ может быть записана в виде:

$$
U_{j}(1) \leqslant\left(c_{4}+c_{5} \omega\right)+c_{5} \omega \zeta_{1}\left(t_{j}\right)^{\frac{q(p+1)}{p-q+1}} \quad \forall j \in \mathbb{N}
$$

где

$$
c_{4}=c_{1} g_{0} T^{\frac{q+1}{p-q}} h_{0}(1), \quad c_{5}=c_{3}\left(1-\lambda^{\frac{1}{1+\mu}}\right)^{-1} .
$$

Так как $h_{0}(s)=0 \forall s>d$, то неравенства (4.7), (4.8) порождают следующую систему:

$$
\begin{array}{ll}
U_{j}(s) \leqslant \lambda_{1} U_{j-1}(s)+r_{5}\left(-U_{j}^{\prime}(s)\right)^{1+\mu} & \forall s>d, \quad \forall j \in \mathbb{N} \\
U_{j}(d) \leqslant\left(c_{4}+c_{5} \omega\right)+c_{5} \omega \zeta_{1}\left(t_{j}\right)^{\frac{q(p+1)}{p-q+1}} \quad \forall j \in \mathbb{N}, \quad \lambda_{1}=\lambda^{\frac{1}{1+\mu}} .
\end{array}
$$

Из этой системы в силу леммы 6.1 (см. §6) вытекает следуюшая равномерная оценка носителей функций $U_{j}(s)$ :

$$
\zeta\left(t_{j}\right) \leqslant \sup \left\{s: s \in \operatorname{supp} U_{j}\right\} \leqslant c_{6}\left[c_{4}+c_{5} \omega+c_{5} \omega \zeta_{1}\left(t_{j}\right)^{\frac{q(p+1)}{p-q+1}}\right]^{\frac{\mu}{1+\mu}}+d,
$$

где $c_{6}=\left(\frac{r_{5}}{1-\lambda_{1}}\right)^{\frac{1}{1+\mu}} \cdot \frac{1+\mu}{\mu}$. Из последнего неравенства вытекает, наконец,

$$
\zeta\left(t_{j}\right) \leqslant d+c_{6}\left(c_{4}+c_{5} \omega\right)^{\frac{\mu}{1+\mu}}+c_{6} c_{5}^{\frac{\mu}{1+\mu}} \omega^{\frac{\mu}{1+\mu}} \zeta_{1}\left(t_{j}\right)^{\varkappa} \quad \forall j \in \mathbb{N},
$$


где

$$
\varkappa=\frac{q(p+1)(p-q)}{(p-q+1)[n(p-q)+(q+1)(p+1)]}<1 \quad \forall n \geqslant 1, \quad p>q .
$$

Очевидно, что для доказательства теоремы 1 достаточно установить оценку сверху для функции $\zeta(t)$. Очевидно также, что такую оценку достаточно установить на множестве

$$
S=\left\{t \in(0, T): \zeta(t)=\zeta_{1}(t)\right\}
$$

$\mathrm{C}$ другой стороны, легко проверить, что существует число $T_{0}<T$, зависящее лишь от параметров $\xi_{1}, \xi_{2}$ из (4.1), такое, что любое число $\tilde{t} \in\left(T_{0}, T\right)$ может быть представлено как некоторая точка из соответствующей последовательности $\left\{t_{j}\right\}$, удовлетворяющей ограничениям (4.1), (4.2), причем $t_{0}=0$. Возьмем теперь произвольную точку $\tilde{t}$ :

$$
\tilde{t} \in\left[T_{0}, T\right) \cap S, \quad \tilde{t}=t_{j_{0}}, \quad j_{0} \in \mathbb{N} .
$$

Тогда в силу установленного соотношения (4.9) и определения (4.10) имеем:

$$
\begin{aligned}
\zeta(\tilde{t})=\zeta\left(t_{j_{0}}\right) & \leqslant d+c_{6}\left(c_{4}+c_{5} \omega\right)^{\frac{\mu}{1+\mu}}+c_{6} c_{5}^{\frac{\mu}{1+\mu}} \zeta(\tilde{t})^{\varkappa} \omega^{\frac{\mu}{\mu+1}} \\
& \leqslant d+c_{6}\left(c_{4}+c_{5} \omega\right)^{\frac{\mu}{1+\mu}}+\varepsilon \zeta(\tilde{t})+c_{7}(\varepsilon)\left(c_{6} c_{5}^{\frac{\mu}{1+\mu}}\right)^{\frac{1}{1-\varkappa} \omega^{\frac{\mu}{(1+\mu)(1-\varkappa)}}}
\end{aligned}
$$

что приводит к оценке:

$$
\begin{gathered}
\zeta(\tilde{t}) \leqslant(1-\varepsilon)^{-1}\left[d+c_{6}\left(c_{4}+c_{5} \omega\right)^{\frac{\mu}{1+\mu}}+c_{7}(\varepsilon)\left(c_{6} c_{5}^{\frac{\mu}{1+\mu}}\right)^{\frac{1}{1-\varkappa}} \omega^{\frac{\mu}{(1+\mu)(1-\varkappa)}}\right] \equiv D(\varepsilon, \omega) \\
D(\varepsilon, 0)=(1-\varepsilon)^{-1}\left(d+c_{6} c_{4}^{\frac{\mu}{1+\mu}}\right)=\frac{D_{0}}{1-\varepsilon} \quad \forall \varepsilon>0
\end{gathered}
$$

Заметим теперь, что все постоянные $c_{i}$, которые фигурировали в вышеприведенных вычислениях, не зависят от внешнего радиуса $R$ области $\Omega$. Поэтому при выполнении условия

$$
d+c_{6} c_{4}^{\frac{\mu}{1+\mu}}<R
$$

можно найти $\varepsilon_{0}>0, \omega=\omega(R)>0$ такие, что

$$
\zeta(\tilde{t})<D(\varepsilon, \omega)<R \quad \forall \tilde{t} \in\left[T_{0}, T\right) .
$$

Эта оценка, как легко видеть, эквивалентна наличию свойства локализации у рассматриваемой граничной задачи при $R$ и $\omega>0$, удовлетворяюших соотношениям $(4.12),(4.13)$. Отметим еще, что $\omega(R) \rightarrow \infty$ при $R \rightarrow \infty$. Теорема 1 доказана.

\section{§5. Условия отсутствия внутренних точек сингулярности решения}

ДоКАЗАТЕЛЬСТво ТЕОРЕмЫ 2 также основывается на рассмотрении свойств бесконечной системы (3.8), (3.9). Будем поэтапно детализировать выбор последовательности $\left\{t_{i}\right\}$. Пока будем считать выполненным только одно ограничение:

$$
\Delta_{i+1} \leqslant \xi_{2} \Delta_{i} \quad \forall i \in \mathbb{N}, \quad \xi_{2}=\text { const }<1
$$


Зафиксируем теперь число $\xi$ :

$$
\frac{v}{1+\mu}<\xi<\frac{\nu}{\mu} \equiv \alpha_{1}=\frac{q+1}{p-q}
$$

и введем нормированные семейства неизвестных функций:

$$
A_{j}(s) \equiv \Delta_{j}^{\xi} E_{j}(s), \quad H_{j}(s)=\Delta_{j}^{\xi} h_{j}(s), \quad j=1,2, \ldots
$$

При этом, как несложно проверить, соотношения (3.8), (3.9) эквивалентны следующим:

$$
\begin{aligned}
& A_{j}(s) \leqslant r_{1}\left(\frac{\Delta_{j}}{\Delta_{j-1}}\right)^{\xi} H_{j-1}(s)+r_{2} \Delta_{j}^{\nu-\xi \mu}\left(-A_{j}^{\prime}(s)\right)^{1+\mu} \quad \forall s>1, \\
& H_{j}(s) \leqslant\left(1+\delta_{j}\right)\left(\frac{\Delta_{j}}{\Delta_{j-1}}\right)^{\xi} H_{j-1}(s)+r_{3} \delta_{i}^{-\frac{(p+1) \nu}{q+1}} \Delta_{j}^{\nu-\varepsilon \mu}\left(-A_{j}^{\prime}(s)\right)^{1+\mu} \quad \forall s>1 .
\end{aligned}
$$

Зафикисируем число $\lambda, 1>\lambda>\xi_{2}^{\xi}$, и определим $\delta_{j}$ :

$$
\delta_{j}=\delta_{0}=\lambda \xi_{2}^{-\xi}-1 \quad \forall j \in \mathbb{N} .
$$

Тогда в силу (5.1)

$$
\left(1+\delta_{0}\right)\left(\frac{\Delta_{j}}{\Delta_{j-1}}\right)^{\xi} \equiv \lambda_{j}<\lambda<1 \quad \forall j \in \mathbb{N} .
$$

Теперь стартовые неравенства примут вид:

$$
\begin{aligned}
& A_{j}(s) \leqslant r_{4} \lambda_{j} H_{j-1}(s)+r_{2} \Delta_{j}^{\nu-\xi \mu}\left(-A_{j}^{\prime}(s)\right)^{1+\mu}, \quad r_{4}=r_{1}\left(1+\delta_{0}\right)^{-1} \\
& H_{j}(s) \leqslant \lambda_{j} H_{j-1}(s)+r_{5} \Delta_{j}^{\nu-\xi \mu}\left(-A_{j}^{\prime}(s)\right)^{1+\mu}, \quad r_{5}=r_{3} \delta_{0}^{-\frac{\nu(p+1)}{q+1}} .
\end{aligned}
$$

Итерируем неравенство (5.4), учитывая при этом оценку (5.5), и получаем:

$$
\begin{aligned}
A_{j}(s) \leqslant & r_{4} \prod_{i=1}^{j} \lambda_{i} H_{0}(s)+r_{4} r_{5}\left[\lambda_{j} \lambda_{j-1} \cdots \lambda_{2} \Delta_{1}^{\nu-\xi \mu}\left(-A_{1}^{\prime}(s)\right)^{1+\mu}\right. \\
& +\lambda_{j} \cdots \lambda_{3} \Delta_{2}^{\nu-\xi \mu}\left(-A_{2}^{\prime}(s)\right)^{1+\mu} \\
& \left.+\cdots+\lambda_{j} \Delta_{j-1}^{\nu-\xi \mu}\left(-A_{j-1}^{\prime}(s)\right)^{1+\mu}\right] \\
& +r_{2} \Delta_{j}^{\nu-\xi \mu}\left(-A_{j}^{\prime}(s)\right)^{1+\mu}, \quad H_{0}(s) \equiv T^{\xi} h_{0}(s)
\end{aligned}
$$

Так как

$$
\lambda_{j} \lambda_{j-1} \cdots \lambda_{i+1} \Delta_{i}^{\nu-\xi \mu}=\left(1+\delta_{0}\right)^{j-i} \Delta_{j}^{\nu-\xi \mu}\left(\frac{\Delta_{j}}{\Delta_{i}}\right)^{(1+\mu) \xi-\nu}
$$


то из последнего неравенства вытекает соотношение:

$$
\begin{aligned}
& A_{j}(s) \leqslant r_{4}\left(1+\delta_{0}\right)^{j}\left(\frac{\Delta_{j}}{\Delta_{0}}\right)^{\xi} H_{0}(s) \\
&+\left[\sum_{i=1}^{j}\left(1+\delta_{0}\right)^{j-i}\left(\frac{\Delta_{j}}{\Delta_{i}}\right)^{\xi(1+\mu)-\nu}\left(-A_{i}^{\prime}(s)\right)^{1+\mu}\right] r_{6} \Delta_{j}^{\nu-\xi \mu} \\
& \forall j \in \mathbb{N}, \quad \forall s>1 .
\end{aligned}
$$

Введем еще одно семейство функций:

$$
\begin{aligned}
U_{j}(s) & =\sum_{i=1}^{j}\left(1+\delta_{0}\right)^{\frac{j-i}{1+\mu}}\left(\frac{\Delta_{j}}{\Delta i}\right)^{\xi-\frac{\nu}{1+\mu}} A_{i}(s) \\
& \equiv \sum_{i=1}^{j}\left(1+\delta_{0}\right)^{\frac{j-i}{1+\mu}}\left(\frac{\Delta_{j}}{\Delta_{i}}\right)^{\xi-\frac{\nu}{1+\mu}} \Delta_{i}^{\xi} E_{i}(s), \quad j=1,2, \ldots
\end{aligned}
$$

Теперь из соотношений (5.6) вытекает:

$$
A_{j}(s) \leqslant r_{4}\left(1+\delta_{0}\right)^{j}\left(\frac{\Delta_{j}}{\Delta_{0}}\right)^{\xi} H_{0}(s)+r_{6} \Delta_{i}^{\nu-\xi \mu}\left(-U_{j}^{\prime}(s)\right)^{1+\mu} \quad \forall j \in \mathbb{N}
$$

Очевидно следуюшее соотношение:

$$
U_{j}(s)-A_{j}(s)=U_{j-1}(s)\left(1+\delta_{0}\right)^{\frac{1}{1+\mu}}\left(\frac{\Delta_{j}}{\Delta_{j-1}}\right)^{\xi-\frac{\nu}{1+\mu}}
$$

поэтому из неравенства (5.7) вытекает оценка:

$$
\begin{aligned}
& U_{j}(s) \leqslant r_{4}\left(1+\delta_{0}\right)^{j}\left(\frac{\Delta_{j}}{\Delta_{0}}\right)^{\xi} H_{0}(s) \\
&+\left(1+\delta_{0}\right)^{\frac{1}{1+\mu}}\left(\frac{\Delta_{j}}{\Delta_{j-1}}\right)^{\xi-\frac{\nu}{1+\mu}} U_{j-1}(s)+r_{6} \Delta_{j}^{\nu-\xi \mu}\left(-U_{j}^{\prime}(s)\right)^{1+\mu} \\
& \leqslant r_{4} \lambda^{j} H_{0}(s)+\mu_{j} U_{j-1}(s)+r_{6} \Delta_{j}^{\nu-\xi \mu}\left(-U_{j}^{\prime}(s)\right)^{1+\mu} \quad \forall j \in \mathbb{N}, \\
& \mu_{j} \equiv\left(1+\delta_{0}\right)^{\frac{1}{1+\mu}}\left(\frac{\Delta_{j}}{\Delta_{j-1}}\right)^{\xi-\frac{\nu}{1+\mu}} .
\end{aligned}
$$

Дальнейшее рассмотрение распадается на два случая в зависимости от степени сингулярности граничного режима.

1) Случай "пологих" режимов, т.е.

$$
F(t) \leqslant \omega(T-t)^{-\alpha} \quad \forall t<T, \quad \omega=\mathrm{const}<\infty, \quad \alpha<\alpha_{1} \equiv \frac{q+1}{p-q} .
$$


Выбор последовательности $\left\{t_{i}\right\}$ будет связан с нашей основной неизвестной “энергетической” функцией

$$
E(t, s) \equiv \int_{0}^{t} \int_{\Omega(s)}\left|D_{x} u(\tau, x)\right|^{p+1} d x d \tau
$$

Зафиксируем произвольно точку $s^{\prime}>1$ такую, что

$$
E\left(t, s^{\prime}\right) \rightarrow \infty \text { при } t \rightarrow T \text {. }
$$

Если бы такой точки не оказалось, то утверждение теоремы 2 уже было бы "почти" доказано. Поэтому

$$
E\left(t_{j}, s^{\prime}\right) \equiv \sum_{i=1}^{j} E_{i}\left(s^{\prime}\right) \equiv \sum_{i=1}^{j}\left[E\left(t_{i}, s^{\prime}\right)-E\left(t_{i-1}, s^{\prime}\right)\right] \rightarrow \infty
$$

при $j \rightarrow \infty$ в случае любой последовательности $t_{j} \rightarrow T$.

Зафиксируем теперь число $\eta$ так, что

$$
\frac{\nu}{1+\mu}<\xi<\eta<\frac{\nu}{\mu} \equiv \frac{q+1}{p-q}, \quad \alpha<\eta
$$

Наконец, зафиксируем постоянную $g>0$ и определим последовательность $\left\{t_{i}\right\}$ следующим равенством:

$$
\left(t_{j}-t_{j-1}\right)^{-\eta} \equiv \Delta_{j}^{-\eta}=g\left(E\left(t_{j}, s^{\prime}\right)-E\left(t_{j-1}, s^{\prime}\right)\right) \quad \forall j \in \mathbb{N}
$$

Корректность этого определения легко следует из непрерьвности и монотонности функции $E\left(t, s^{\prime}\right)$.

Проверим теперь, что при соответствуюшем выборе постоянной $g$ построенная последовательность будет удовлетворять условию (5.1). Более того, покажем выполнение более сильного неравенства

$$
\Delta_{j} \geqslant(1+\gamma)\left(T-t_{j}\right) \quad \forall j \in \mathbb{N}
$$

с некоторьм единым $\gamma>0$. Допустим, что неравенство (5.12) не имеет места, т.е. существует номер $i$ такой, что

$$
\Delta_{i}<(1+\gamma)\left(T-t_{i}\right) \Longleftrightarrow \Delta_{i}^{-\alpha}>(1+\gamma)^{-\alpha}\left(T-t_{i}\right)^{-\alpha}
$$

Мы находимся сейчас в условиях применимости теоремы 1, поэтому

$$
\zeta_{1}(t) \leqslant \tilde{c}=\text { const }<\infty \quad \forall t<T
$$

и, следовательно, из (3.3) вытекает априорная оценка:

$$
E_{j}(1) \leqslant c_{1} h_{0}(1)+c_{2} F\left(t_{j}\right) \leqslant c_{1} h_{0}(1)+c_{2} \omega\left(T-t_{j}\right)^{-\alpha} \quad \forall j \in \mathbb{N} .
$$


Теперь в силу (5.11), (5.13) и (5.14) имеем:

$$
\Delta_{i}^{-\eta}=g E_{i}\left(s^{\prime}\right) \leqslant g c_{1} h_{0}(1)+g c_{2} \omega\left(T-t_{i}\right)^{-\alpha} \leqslant g c_{1} h_{0}(1)+\omega g c_{2}(1+\gamma)^{\alpha} \Delta_{i}^{-\alpha}
$$

или

$$
1 \leqslant g\left[c_{1} h_{0}(1) \Delta_{i}^{\eta}+c_{2}(1+\gamma)^{\alpha} \omega \Delta_{i}^{\eta-\alpha}\right] \leqslant g\left[c_{1} h_{0}(1) T^{\eta}+c_{2}(1+\gamma)^{\alpha} T^{\eta-\alpha} \omega\right]
$$

Следовательно, предположив заранее, что

$$
g<\left[c_{1} h_{0}(1) T^{\eta}+c_{2} \omega(1+\gamma)^{\alpha} T^{\eta-\alpha}\right]^{-1},
$$

получим противоречие с допушением (5.13).

Итак, имеем последовательность $\left\{\Delta_{i}\right\}$, удовлетворяюшую (5.11) и ограничению $(5.1)$ с $\xi_{2}=(1+\gamma)^{-1}<1$. Соответственно $\delta_{0}>0$ зафиксировано так, что выполнено также неравенство (5.3) и

$$
\mu_{j}=\left(1+\delta_{0}\right)^{\frac{1}{1+\mu}}\left(\frac{\Delta_{j}}{\Delta_{j-1}}\right)^{\eta-\frac{\nu}{1+\mu}}<\mu_{0}<1 \quad \forall j \in \mathbb{N}
$$

Соотношение (5.8) теперь дает

$$
U_{j}(s) \leqslant r_{4} \lambda^{j} H_{0}(s)+\mu_{0} U_{j-1}(s)+r_{6} \Delta_{j}^{\nu-\xi \mu}\left(-U_{j}^{\prime}(s)\right)^{1+\mu} \quad \forall j \in \mathbb{N} .
$$

Кроме того, имеем в силу (5.11) и (5.16):

$$
\begin{aligned}
U_{j}\left(s^{\prime}\right) & =\sum_{i=1}^{j}\left(1+\delta_{0}\right)^{\frac{j-i}{1+\mu}}\left(\frac{\Delta_{j}}{\Delta i}\right)^{\xi-\frac{\nu}{1+\mu}} \Delta_{i}^{\xi} \Delta_{i}^{-\eta} g^{-1} \\
& =g^{-1} \Delta_{j}^{-(\eta-\xi)} \sum_{i=1}^{j}\left(1+\delta_{0}\right)^{\frac{j-i}{1+\mu}}\left(\frac{\Delta_{j}}{\Delta_{i}}\right)^{\eta-\frac{\nu}{1+\mu}} \\
& \leqslant g^{-1} \Delta_{j}^{-(\eta-\xi)} \sum_{i=1}^{j}\left(1+\delta_{0}\right)^{\frac{j-i}{1+\mu}} \prod_{k=i+1}^{j}\left(\frac{\Delta_{k}}{\Delta_{k-1}}\right)^{\eta-\frac{\nu}{1+\mu}} \\
& \leqslant g^{-1} \Delta_{j}^{-(\eta-\xi)} \sum_{i=1}^{j} \mu_{0}^{j-i} \\
& \leqslant g^{-1} \Delta_{j}^{-(\eta-\xi)}\left(1-\mu_{0}\right)^{-1}
\end{aligned}
$$

т.е.

$$
U_{j}\left(s^{\prime}\right) \leqslant g^{-1}\left(1-\mu_{0}\right)^{-1} \Delta_{j}^{-(\eta-\xi)} \quad \forall j \in \mathbb{N} .
$$

Применим теперь к дифференциальной системе (5.17), (5.18) лемму 6.3 из 6 . В результате получим следующую равномерную априорную оценку:

$$
U_{j}(s) \leqslant c \max \left\{U_{0}\left(s-s^{\prime}\right), H_{0}(s)\right\} \quad \forall j \in \mathbb{N}, \quad \forall s>s^{\prime},
$$


где $U_{0}(s)=s^{-\frac{(\eta-\xi)(1+\mu)}{\nu-\xi \mu}}$. Очевидно, легко указать такое $s^{(0)}>0$, что

$$
U_{j}(s) \leqslant c U_{0}\left(s-s^{\prime}\right) \quad \forall s: s^{\prime}<s<s^{\prime}+s^{(0)},
$$

и, следовательно, в силу определения (5.11) имеем:

$$
\begin{gathered}
E\left(t_{j}, s\right)-E\left(t_{j-1}, s\right) \equiv E_{j}(s) \leqslant c U_{0}\left(s-s^{\prime}\right) \Delta_{j}^{-\xi}=c g^{\frac{\xi}{\eta}} U_{0}\left(s-s^{\prime}\right) E_{j}\left(s^{\prime}\right)^{\frac{\xi}{\eta}} \\
\equiv c g^{\frac{\xi}{\eta}} U_{0}\left(s-s^{\prime}\right)\left(E\left(t_{j}, s^{\prime}\right)-E\left(t_{j-1}, s^{\prime}\right)\right)^{\frac{\xi}{\eta}} \\
\forall s \in\left(s^{\prime}, s^{\prime}+s^{(0)}\right), \quad j=1,2, \ldots, \quad E\left(t_{0}, s\right) \equiv 0 .
\end{gathered}
$$

Теперь получим оценку типа (5.20) для $E\left(t, s^{\prime}\right)$ в произвольной точке $t \in(0, T)$. Определим отображение

$$
\Gamma:[0, T) \rightarrow(0, T)
$$

следующим равенством:

$$
(\Gamma(t)-t)^{-\eta}=g\left(E\left(\Gamma(t), s^{\prime}\right)-E\left(t, s^{\prime}\right)\right) \quad \forall t \in(0, T),
$$

где $g>0$ из определения $(5.11), E\left(t, s^{\prime}\right)$ - “энергетическая” функция рассматриваемого решения $u(t, x), s^{\prime}=$ const.

Очевидно, что монотонная последовательность, определяемая форомулой (5.11), есть последовательность, задаваемая рекуррентньм соотношением:

$$
t_{j+1}=\Gamma\left(t_{j}\right) \quad \forall j \in \mathbb{N}, \quad t_{0}=0 .
$$

Из непрерьвности и монотонности функции $E\left(t, s^{\prime}\right)$ по $t$ и из сделанного допущения о том, что $E\left(t, s^{\prime}\right) \rightarrow \infty$ при $t \rightarrow T$, легко выводится непрерывность и строгое монотонное возрастание функции $\Gamma(t)$ на всем интервале $(0, T)$. Следовательно, функция $\Gamma(t)$ является непрерывным монотонньм взаимнооднозначным отображением отрезка $\left[t_{i-1}, t_{i}\right]$ на $\left[t_{i}, t_{i+1}\right] \forall i \in \mathbb{N}$.

Пусть теперь $t$-произвольная точка из интервала $\left[t_{1}, T\right)$. Пусть для определенности $t \in\left(t_{j-1}, t_{j}\right]$ при некотором конкретном $j \in \mathbb{N}$. Тогда, очевидно, единственным образом находится последовательность $\left\{\tilde{t}_{i}\right\}$ такая, что

$$
\tilde{t}_{1} \in\left(t_{0}, t_{1}\right], \quad \tilde{t}_{i+1}=\Gamma\left(\tilde{t}_{i}\right), \quad i=1,2, \ldots, j-1, \quad \tilde{t}_{j}=t .
$$

Относительно расположения точки $\tilde{t}_{1}$ возможны два варианта:

a) $\tilde{t}_{1} \geqslant\left(1+\xi_{2}\right)^{-1} T$,

b) $0<\tilde{t}_{1}<\left(1+\xi_{2}\right)^{-1} T$.

В случае а) последовательность $\left\{\tilde{t}_{i}\right\}, i=1,2, \ldots, j, \tilde{t}_{0}=0$, удовлетворяет всем условиям, необходимым для получения оценок $(5.19),\left(5.19^{*}\right)$ и, следовательно,

$$
\begin{gathered}
E\left(\tilde{t}_{i}, s\right)-E\left(\tilde{t}_{i-1}, s\right) \leqslant c g^{\frac{\xi}{\eta}} U_{0}\left(s-s^{\prime}\right)\left(E\left(\tilde{t}_{i}, s^{\prime}\right)-E\left(\tilde{t}_{i-1}, s^{\prime}\right)\right)^{\frac{\xi}{\eta}} \\
\forall s \in\left(s^{\prime}, s^{\prime}+s^{(0)}\right), \quad i=2,3, \ldots, j ; \\
E\left(\tilde{t}_{1}, s\right) \leqslant c U_{0}\left(s-s^{\prime}\right)\left(1+\xi_{2}\right)^{\xi} T^{-\xi} \quad \forall s \in\left(s^{\prime}, s^{\prime}+s^{(0)}\right) .
\end{gathered}
$$


Складывая все эти неравенства, находим:

$$
\begin{aligned}
E\left(\tilde{t}_{j}, s\right) & \equiv E(t, s) \\
& \leqslant c U_{0}\left(s-s^{\prime}\right)\left[g^{\frac{\xi}{\eta}}\left(E\left(\tilde{t}_{j}, s^{\prime}\right)-E\left(\tilde{t}_{j-1}, s^{\prime}\right)\right)^{\frac{\xi}{\eta}} \sum_{i=2}^{j} \xi_{2}^{(j-i) \xi}+\left(1+\xi_{2}\right)^{\xi} T^{-\xi}\right] \\
& \leqslant c U_{0}\left(s-s^{\prime}\right)\left[g^{\frac{\xi}{\eta}} E\left(t, s^{\prime}\right)^{\frac{\xi}{\eta}}\left(1-\xi_{2}^{\xi}\right)^{-1}+\left(1+\xi_{2}\right)^{\xi} T^{-\xi}\right] \\
& \leqslant c_{1} U_{0}\left(s-s^{\prime}\right)\left(T^{-\xi}+E\left(t, s^{\prime}\right)^{\frac{\xi}{\eta}}\right) .
\end{aligned}
$$

В случае b) имеем в силу (3.3):

$$
\begin{aligned}
h\left(\tilde{t}_{1}, 1\right)+E\left(\tilde{t}_{1}, 1\right) & \equiv \int_{\Omega}\left|u\left(\tilde{t}_{1}, x\right)\right|^{q+1} d x+\int_{0}^{\tilde{t}_{1}} \int_{\Omega}\left|D_{x} u\right|^{p+1} d x d t \\
& \leqslant c_{1} h_{0}(1)+c_{2} F\left(\tilde{t}_{1}\right) \leqslant c_{1} h_{0}(1)+c_{2} \omega\left(T-\tilde{t}_{1}\right)^{-\alpha} \\
& \leqslant c_{1} h_{0}(1)+c_{2} \omega \frac{\left(1+\xi_{2}\right)^{\alpha}}{\xi_{2}^{\alpha}} T^{-\alpha}
\end{aligned}
$$

поэтому, рассматривая $u(t, x)$ как решение задачи $(2.1)-(2.2)$ в области $\left(\tilde{t}_{1}, T\right) \times \Omega$ с начальным условием

$$
u\left(\tilde{t}_{1}, x\right)=u_{1}(x)
$$

где $u_{1}$ - след рассматриваемого решения задачи (2.1)-(2.3) на гиперплоскости $t=\tilde{t}_{1}$, получим аналогично (5.20):

$$
\begin{gathered}
E\left(\tilde{t}_{i}, s\right)-E\left(\tilde{t}_{i-1}, s\right) \leqslant c g^{\frac{\xi}{\eta}} U_{0}\left(s-s^{\prime}\right)\left(E\left(\tilde{t}_{i}, s^{\prime}\right)-E\left(\tilde{t}_{i-1}, s^{\prime}\right)\right)^{\frac{\xi}{\eta}} \\
\forall s \in\left(s^{\prime}, s^{\prime}+s^{(1)}\right), \quad i=2,3, \ldots, j, \quad s^{(1)} \equiv s^{(1)}\left(h\left(\tilde{t}_{1}, 1\right)\right) .
\end{gathered}
$$

Складывая все эти неравенства, а также (5.23), получаем:

$$
\begin{aligned}
E(t, s) \equiv E\left(\tilde{t}_{j}, s\right) \leqslant & c U_{0}\left(s-s^{\prime}\right) g^{\frac{\xi}{\eta}}\left(1-\xi_{2}^{\xi}\right)^{-1}\left(E\left(\tilde{t}_{j}, s^{\prime}\right)-E\left(\tilde{t}_{j-1}, s^{\prime}\right)\right)^{\frac{\xi}{\eta}} \\
& +c_{1} h_{0}(1)+c_{2} \omega \frac{\left(1+\xi_{2}\right)^{\alpha}}{\xi_{2}^{\alpha}} T^{-\alpha} \\
\leqslant & c_{1} U_{0}\left(s-s^{\prime}\right)\left(E\left(t, s^{\prime}\right)^{\frac{\xi}{\eta}}+T^{-\alpha}\right) \quad \forall s \in\left(s^{\prime}, s^{\prime}+s^{(1)}\right) .
\end{aligned}
$$

Теперь доказательство ограниченности функции $E(t, s)$ в любой области вида $(0, T) \times\left(s^{\prime}, R\right) \forall s^{\prime}>1$ проведем методом от противного, т.е. предположим, что существует интервал $\left(1, s^{\prime}\right)$ такой, что

$$
E\left(t_{j}, s^{\prime}\right) \equiv R_{j} \rightarrow \infty \text { при } t_{j} \rightarrow T .
$$

Зафиксируем произвольно номер $j$ и при $t=t_{j}$ рассмотрим установленные оценки (5.22), (5.24). Имеем:

$$
E\left(t_{j}, s\right) \leqslant U_{0}(s-v) E\left(t_{j}, v\right)^{\theta} \quad \forall s, v, \quad 1<v<s<s^{\prime} ; \quad \theta=\frac{\xi}{\eta}<1 .
$$


Введем теперь убывающую последовательность точек $\left\{s_{i}\right\}$ :

$$
s_{i}=1+\left(s^{\prime}-1\right) 2^{-(i-1)}, \quad i=1,2, \ldots, \quad s_{1}=s^{\prime}, \quad s_{i}>1 \quad \forall i \in \mathbb{N} .
$$

В силу (5.26) теперь имеем серию соотношений:

$$
E\left(t_{j}, s_{i}\right) \leqslant U_{0}\left(s_{i}-s_{i+1}\right) E\left(t_{j}, s_{i+1}\right)^{\theta} \quad \forall i \in \mathbb{N} .
$$

Итерируя последнее неравенство, получаем:

$$
\begin{aligned}
E\left(t_{j}, s^{\prime}\right) \equiv E\left(t_{j}, s_{1}\right) & \leqslant U_{0}\left(s_{1}-s_{2}\right) E\left(t_{j}, s_{2}\right)^{\theta} \\
& \leqslant U_{0}\left(s_{1}-s_{2}\right) U_{0}\left(s_{2}-s_{3}\right)^{\theta} E\left(t_{j}, s_{3}\right)^{\theta^{2}} \\
& \leqslant \cdots \leqslant \prod_{i=1}^{k} U_{0}\left(s_{i}-s_{i+1}\right)^{\theta^{i-1}} E\left(t_{j}, s_{k+1}\right)^{\theta^{k}} .
\end{aligned}
$$

Следовательно,

$$
E\left(t_{j}, s^{\prime}\right) \leqslant \lim _{k \rightarrow \infty} \prod_{i=1}^{k} U_{0}\left(s_{i}-s_{i+1}\right)^{\theta^{i-1}} E\left(t_{j}, s_{k+1}\right)^{\theta^{k}}
$$

Так как

$$
E\left(t_{j}, s_{k+1}\right) \leqslant E\left(t_{j}, 1\right) \leqslant N_{j}<\infty \quad \forall j \in \mathbb{N},
$$

то в силу того, что $\theta<1$, следует

$$
\lim _{k \rightarrow \infty} E\left(t_{j}, s_{k+1}\right)^{\theta^{k}} \leqslant \lim _{k \rightarrow \infty} N_{j}^{\theta^{k}}=1 \quad \forall j \in \mathbb{N} .
$$

Теперь в силу определения функции $U_{0}$ имеем:

$$
\begin{aligned}
U_{0}\left(s_{i}-s_{i+1}\right) & \leqslant c\left(s_{i}-s_{i+1}\right)^{-\frac{(\eta-\xi)(1+\mu)}{\nu-\xi \mu}} \equiv c\left(s_{i}-s_{i+1}\right)^{-\beta} \\
& =c\left(s^{\prime}-1\right)^{-\beta} 2^{i \beta}=c_{1} 2^{i \beta} \quad \forall i \in \mathbb{N} .
\end{aligned}
$$

Поэтому

$$
\begin{aligned}
\ln \left(\prod_{i=1}^{\infty} U_{0}\left(s_{i}-s_{i+1}\right)^{\theta^{i-1}}\right) & =\sum_{i=1}^{\infty} \theta^{i-1} \ln \left(U_{0}\left(s_{i}-s_{i+1}\right)\right) \\
& \leqslant \sum_{i=1}^{\infty} \theta^{i-1}\left(\ln c_{1}+i \beta \ln 2\right) \\
& =\ln c_{1} \sum_{i=1}^{\infty} \theta^{i-1}+\beta \ln 2 \sum_{i=1}^{\infty} i \theta^{i-1} \leqslant c_{2}<\infty
\end{aligned}
$$

Поэтому из (5.28) вытекает оценка:

$$
E\left(t_{j}, s^{\prime}\right) \leqslant \exp c_{2} \quad \forall j \leqslant \mathbb{N}
$$


где, очевидно, $c_{2}<\infty$ не зависит от номера $j$. Эта оценка противоречит допущению (5.25). Следовательно, ограниченность функции $E(t, s)$ в произвольной области вида $(0, T) \times\left(s^{\prime}, R\right) \forall s^{\prime}>1$ при граничном режиме (5.9) доказана.

2) Случай граничного режима, близкого к критическому, т.е.

$$
F(t) \leqslant \widetilde{\omega}(t)(T-t)^{-\alpha_{1}} \quad \forall t<T, \quad \alpha_{1}=\frac{q+1}{p-q}
$$

где $\widetilde{\omega}(t)$ - произвольная непрерывная функция, удовлетворяющая лишь одному условию

$$
\lim _{t \rightarrow T} \widetilde{\omega}(t)=0
$$

В этом случае определяем последовательность $\left\{t_{i}\right\}$ равенством:

$$
t_{i+1}-t_{i} \equiv \Delta_{i+1}=\xi_{2} \Delta_{i}=\xi_{2}\left(t_{i}-t_{i-1}\right) \quad \forall i \geqslant 2, \quad t_{1}=\left(1-\xi_{2}\right) T
$$

Очевидно, что

$$
\sum_{i=1}^{\infty} \Delta_{i}=T
$$

Указанная последовательность удовлетворяет условию (5.1), поэтому для вьше введенных функций $U_{j}(s)$ выполняется система неравенств (5.17). Но оценку начального значения $U_{j}(1)$ мы сейчас проведем иначе, чем в случае 1$)$.

$$
U_{j}(1)=\sum_{i=1}^{j}\left(1+\delta_{0}\right)^{\frac{j-i}{1+\mu}}\left(\frac{\Delta_{j}}{\Delta_{i}}\right)^{\xi-\frac{\nu}{1+\mu}} \Delta_{i}^{\xi} E_{i}(1) \quad \forall j \in \mathbb{N} .
$$

В силу (5.14) и (5.32) имеем оценку для $E_{i}(1)$ :

$$
\begin{aligned}
E_{i}(1) & \leqslant c_{1} h_{0}(1)+c_{2} F\left(t_{i}\right) \leqslant c_{1} h_{0}(1)+c_{2} \widetilde{\omega}_{i}\left(T-t_{i}\right)^{-\alpha_{1}} \\
& =c_{1} h_{0}(1)+c_{2} \xi_{2}^{\alpha_{1}}\left(1-\xi_{2}\right)^{-\alpha_{1}} \Delta_{i}^{-\alpha_{1}} \quad \forall i \in \mathbb{N}, \quad \tilde{\omega}_{i} \equiv \widetilde{\omega}\left(t_{i}\right) .
\end{aligned}
$$

Поэтому из (5.33) следует:

$$
U_{j}(1) \leqslant c_{3} h_{0}(1)+c_{4} \sum_{i=1}^{j}\left(1+\delta_{0}\right)^{\frac{j-i}{1+\mu}}\left(\frac{\Delta_{j}}{\Delta_{i}}\right)^{\xi-\frac{\nu}{1+\mu}} \Delta_{i}^{\xi-\alpha_{1}} \widetilde{\omega}_{i}
$$

где

$$
\begin{aligned}
c_{3} & \leqslant c_{1} \sum_{i=1}^{j}\left(\prod_{k=i}^{j-1}\left(1+\delta_{0}\right)^{\frac{1}{1+\mu}}\left(\frac{\Delta_{k+1}}{\Delta_{k}}\right)^{\xi-\frac{\nu}{1+\mu}}\right) \Delta_{i}^{\xi} \leqslant c_{1} \sum_{i=1}^{j} \mu_{0}^{j-i} \Delta_{i}^{\xi} \\
& \leqslant c_{1} \Delta_{1}^{\xi}\left(1-\mu_{0}\right)^{-1} \leqslant c_{1}\left(1-\mu_{0}\right)^{-1} T^{\xi}, \quad c_{4}=c_{2} \xi_{2}^{\alpha_{1}}\left(1-\xi_{2}\right)^{-\alpha_{1}}
\end{aligned}
$$

Пусть $\left\{\omega_{i}\right\}, i=1,2, \ldots,-$ произвольная последовательность, мажорирующая последовательность $\left\{\widetilde{\omega}_{i}\right\}$ :

$$
\omega_{i} \geqslant \widetilde{\omega}_{i} \forall i \in \mathbb{N}, \quad \omega_{i} \rightarrow 0 \text { при } i \rightarrow \infty
$$


и удовлетворяющая дополнительному условию правильности:

$$
\omega_{i+1} \geqslant \omega_{i} \mu_{0} \xi_{2}^{\alpha_{1}-\xi} \chi^{-1} \quad \forall i \in \mathbb{N}
$$

где $\chi, 0<\chi<1,-$ произвольное фиксированное число.

Так как $\mu_{0} \xi_{2}^{\alpha_{1}-\xi}<1$, то, как несложно убедиться, найти такое $\chi<1$ и последовательность $\left\{\omega_{i}\right\}$ можно для любой исходной последовательности $\left\{\widetilde{\omega}_{i}\right\}, \widetilde{\omega}_{i} \rightarrow 0$ при $i \rightarrow \infty$.

Вернемся теперь снова к неравенству (5.35). В силу (5.36), (5.37) имеем:

$$
\begin{aligned}
& \sum_{i=1}^{j}\left(1+\delta_{0}\right)^{\frac{j-i}{1+\mu}}\left(\frac{\Delta_{j}}{\Delta_{i}}\right)^{\xi-\frac{\nu}{1+\mu}} \Delta_{i}^{\xi-\alpha_{1}} \widetilde{\omega}_{i} \\
& \quad \leqslant \omega_{j} \Delta_{j}^{-\left(\alpha_{1}-\xi\right)} \sum_{i=1}^{j}\left(1+\delta_{0}\right)^{\frac{j-i}{1+\mu}}\left(\frac{\Delta_{j}}{\Delta_{i}}\right)^{\alpha_{1}-\frac{\nu}{1+\mu}} \frac{\omega_{i}}{\omega_{j}} \\
& \quad \leqslant \omega_{j} \Delta_{j}^{-\left(\alpha_{1}-\xi\right)} \sum_{i=1}^{j}\left(1+\delta_{0}\right)^{\frac{j-i}{1+\mu}} \xi_{2}^{(j-i)\left(\alpha_{1}-\frac{\nu}{1+\mu}\right)} \frac{\omega_{i}}{\omega_{j}} \\
& \quad \leqslant \omega_{j} \Delta_{j}^{-\left(\alpha_{1}-\xi\right)} \sum_{i=1}^{j}\left[\left(1+\delta_{0}\right)^{\frac{1}{1+\mu}} \xi_{2}^{\xi-\frac{\nu}{1+\mu}} \xi_{2}^{\alpha_{1}-\xi} \chi \xi_{2}^{\xi-\alpha_{1}} \mu_{0}^{-1}\right]^{j-i} \\
& \leqslant \omega_{j} \Delta_{j}^{-\left(\alpha_{1}-\xi\right)} \sum_{i=1}^{j} \chi^{j-i} \leqslant(1-\chi)^{-1} \omega_{j} \Delta_{j}^{-\left(\alpha_{1}-\xi\right)} .
\end{aligned}
$$

Поэтому, продолжая (5.35), получаем:

$$
U_{j}(1) \leqslant c_{3} h_{0}(1)+c_{4}(1-\chi)^{-1} \omega_{j} \Delta_{j}^{-\left(\alpha_{1}-\xi\right)} .
$$

Теперь рассмотрим дифференциальную систему (5.17), (5.38). Так как

$$
\left[\omega_{j} \Delta_{j}^{-\left(\alpha_{1}-\xi\right)}\right]^{\mu} \Delta_{j}^{\nu-\xi \mu}=\omega_{j}^{\mu} \rightarrow 0 \text { при } j \rightarrow \infty
$$

то к указанной системе применима лемма 6.2 из $\S 6$ и, следовательно, сушествует некоторая функция $U_{0}(s): U_{0}(s)<\infty \forall s>1, U_{0}(s) \rightarrow \infty$ при $s \rightarrow 1$, такая, что справедлива следуюшая равномерная априорная оценка:

$$
U_{j}(s) \leqslant U_{0}(s) \quad \forall j \in \mathbb{N}, \quad \forall s>1
$$

Следовательно,

$$
E_{j}(s)=E\left(t_{j}, s\right)-E\left(t_{j-1}, s\right) \leqslant c U_{0}(s) \Delta_{j}^{-\xi} \quad \forall j \in \mathbb{N} .
$$

Суммируя эти неравенства, получаем:

$$
E\left(t_{j}, s\right) \leqslant c U_{0}(s)\left(1-\xi_{2}^{\xi}\right)^{-1} \Delta_{j}^{-\xi}=\tilde{c} U_{0}(s)\left(T-t_{j}\right)^{-\xi},
$$

где $\tilde{c}=c\left(1-\xi_{2}^{\xi}\right)^{-1}\left(1-\xi_{2}\right)^{-\xi} \xi_{2}^{\xi}$. 
Из последней оценки окончательно следует:

$$
E(t, s) \leqslant c^{\prime} U_{0}(s)(T-t)^{-\xi} \quad \forall t<T, \quad c^{\prime}=\tilde{c}\left(1-\xi_{2}\right)^{-\xi} .
$$

Теперь мы готовы закончить рассуждения для случая произвольного "пологого" граничного режима, удовлетворяющего условию (5.31). Для любого сколь угодно малого $\delta>0$ энергетическая функция $E(t, 1+\delta)$ удовлетворяет, как это следует из (5.40), степенной оценке на рост:

$$
E(t, 1+\delta) \leqslant \omega(T-t)^{-\xi} \quad \forall t<T, \quad \omega=\omega(\delta)=c^{\prime} U_{0}(1+\delta),
$$

где $\xi<(q+1) /(p-q)$. Поэтому, проводя рассуждения из части 1$)$, устанавливаем, что

$$
E(t, s)<\mathrm{const}=c(\delta)<\infty \quad \forall(t, s) \in(0, T) \times(1+2 \delta, R) .
$$

Так как $\delta>0$ может быть выбрано сколь угодно малым, то приходим к требуемой ограниченности функции $E(t, s)$. Теперь оценка

$$
M(t, s) \equiv \int_{(0, t) \times \Omega(s)}|u(\tau, x)|^{p+1} d x d \tau \leqslant c=c(s)<\infty \quad \forall t \leqslant T, \quad \forall s>1
$$

получается благодаря неравенству Пуанкаре. Наконец, из соотношения (3.7) при $a=0$ вытекает неравенство:

$$
\begin{aligned}
N(b, s) & \equiv \int_{\Omega(s+\delta)}|u(b, x)|^{q+1} d x \\
& \leqslant h_{0}(s)+c \delta^{-1} E(b, s)^{\frac{p}{p+1}} M(b, s)^{\frac{1}{p+1}} \quad \forall s>0, \quad \forall \delta>0 .
\end{aligned}
$$

Отсюда, очевидно, следует, что

$$
N(t, s) \leqslant c=c(s)<\infty \quad \forall t \leqslant T, \quad \forall s>1 .
$$

Теорема 2 доказана полностью.

\section{§6. Приложение}

ЛЕмма 6.1. Пусть некоторое семейство непрерьвно дифферениируемых неотрицательных невозрастающих на интервале $[0, \infty)$ функиий $\left\{U_{i}(s)\right\}$, $i=1,2, \ldots, j, j \leqslant \infty$, удовлетворяет следующей системе дифференииальньх неравенств:

$$
\begin{gathered}
U_{i}(s) \leqslant \lambda U_{i-1}(s)+k\left(-U_{i}^{\prime}(s)\right)^{1+\gamma} \quad \forall s \in(0, \infty), \quad 0<\lambda<1, \quad U_{0}(s) \equiv 0, \\
U_{i}(0) \leqslant K_{i}<\infty \quad \forall i \leqslant j, \quad k=\mathrm{const}<\infty, \quad \gamma=\mathrm{const}>0,
\end{gathered}
$$

где $\left\{K_{i}\right\}$ - неубивающая последовательность положсительных чисел. Тогда для указанных функиий $U_{i}(s)$ справедливы следующие априорнье оценки:

$$
U_{i}(s) \leqslant M_{i}(s) \equiv a_{1} k^{-\frac{1}{\gamma}}\left[a_{2}\left(k K_{i}^{\gamma}\right)^{\frac{1}{1+\gamma}}-s\right]_{+}^{\frac{1+\gamma}{\gamma}} \quad \forall i \leqslant j, \quad \forall s>0,
$$

$2 \partial e$

$a_{1}=(1-\lambda)^{\frac{1}{\gamma}}\left(\frac{\gamma}{1+\gamma}\right)^{\frac{1+\gamma}{\gamma}}, \quad a_{2}=(1+\gamma) \gamma^{-1}(1-\lambda)^{-\frac{1}{1+\gamma}}, \quad f(s)_{+} \equiv \max (0, f(s))$. В частности, справедливь следующие оценки для носителей всех функций $U_{i}(s)$ :

$$
\operatorname{supp} U_{i} \in\left[0, b_{i}\right], \quad b_{i}=a_{2}\left(k K_{i}^{\gamma}\right)^{\frac{1}{1+\gamma}}
$$


ДокАЗАТЕльство. Указанные выше функции $M_{i}(s)$ являются, как несложно проверить, решениями следуюших вспомогательных задач Коши:

$$
\begin{gathered}
M_{i}(s)=\lambda M_{i}(s)+k\left(-M_{i}^{\prime}(s)\right)^{1+\gamma} \quad \forall s>0, \\
M_{i}(0)=K_{i} \quad \forall i \leqslant j .
\end{gathered}
$$

Докажем теперь оценку (6.3) по индукции. При $i=1$ эта оценка проверяется непосредственно. Предположим, что (6.3) доказано уже для всех $i \leqslant j-1$, т.е.

$$
0 \leqslant U_{i}(s) \leqslant M_{i}(s), \quad i=1,2, \ldots, j-1,
$$

и докажем теперь, что

$$
U_{j}(s) \leqslant M_{j}(s) \quad \forall s>0 .
$$

Доказательство (6.8) проведем от противного, т.е. предположим, что (6.8) не имеет места. Тогда найдется интервал $\left(s_{1}, s_{2}\right)$ такой, что

$$
U_{j}(s)>M_{j}(s)>0 \quad \forall s \in\left(s_{1}, s_{2}\right), \quad U_{j}\left(s_{1}\right) \equiv M_{j}\left(s_{1}\right) .
$$

Из определения функций $M_{i}(s)$ и предположения индукции (6.7) следует, что

$$
M_{j}(s) \geqslant M_{j-1}(s) \geqslant U_{j-1}(s) \quad \forall s>0 .
$$

В силу этого неравенства и предположения (6.9) теперь выполнено соотношение:

$$
U_{j}(s) \geqslant U_{j-1}(s) \quad \forall s \in\left(s_{1}, s_{2}\right) .
$$

И значит, $U_{j}(s)$ является решением следуюшей задачи:

$$
\begin{gathered}
U_{j}(s) \leqslant \lambda U_{j}(s)+k\left(-U_{j}^{\prime}(s)\right)^{1+\gamma} \quad \forall s \in\left(s_{1}, s_{2}\right), \\
U_{j}\left(s_{1}\right)=M_{j}\left(s_{1}\right) .
\end{gathered}
$$

Интегрируя теперь непосредственно неравенство (6.10), легко приходим к оценке:

$$
U_{j}(s) \leqslant M_{j}(s) \quad \forall s \in\left(s_{1}, s_{2}\right),
$$

что противоречит сделанному допушению (6.9), и значит, утверждение леммы 6.1 доказано.

ЛЕМма 6.2. Пусть некоторое семейство неотрицательных непрерьвно дифференцируемых невозрастающих на интервале $[0, \infty)$ функиий $\left\{U_{i}(s)\right\}$, $i=1,2, \ldots$, удовлетворяет следующей дифференциальной системе:

$$
\begin{gathered}
U_{i}(s) \leqslant a+\lambda U_{i-1}(s)+k_{i}\left(-U_{i}^{\prime}(s)\right)^{1+\gamma} \quad \forall s>0, \quad i=2,3, \ldots, \\
U_{1}(s) \leqslant a+k_{1}\left(-U_{1}^{\prime}(s)\right)^{1+\gamma}, \\
U_{i}(0) \leqslant K_{i}, \quad 0<\lambda<1, \quad a=\mathrm{const}<\infty, \quad \gamma>0,
\end{gathered}
$$

әде $\left\{k_{i}\right\}$ - монотонно убьвающая последовательность, $\left\{K_{i}\right\}$-монотонно возрастающая последовательность: $k_{i} \rightarrow 0, K_{i} \rightarrow \infty$ при $i \rightarrow \infty$, причем

$$
k_{i} K_{i}^{\gamma} \rightarrow 0 \text { монотонно } \quad \text { при } i \rightarrow \infty
$$

Тогда существует непрерьвная невозрастающая на интервале $(0, \infty)$ функция $U_{0}(s)$ такая, что $U_{0}(s) \rightarrow \infty$ при $s \rightarrow 0$ и для всего семейства $\left\{U_{i}(s)\right\}$ выполняется равномерная априорная оченка:

$$
U_{i}(s) \leqslant U_{0}(s), \quad i=1,2, \ldots, \quad \forall s>0 .
$$


ДокАЗАтЕльство. Снова запишем последовательность вспомогательњых задач Коши:

$$
\begin{gathered}
(1-\lambda) M_{i}(s)=a+k_{i}\left(-M_{i}^{\prime}(s)\right)^{1+\gamma} \quad \forall s>0 \\
M_{i}(0)=K_{i}, \quad i=1,2, \ldots
\end{gathered}
$$

Легко проверить, что решениями этих задач являются функции:

$$
M_{i}(s)=\frac{a}{1-\lambda}+a_{1} k_{i}^{-\frac{1}{\gamma}}\left[a_{2}\left(k_{i}\left(K_{i}-\frac{a}{1-\lambda}\right)^{\gamma}\right)^{\frac{1}{1+\gamma}}-s\right]_{+}^{\frac{1+\gamma}{\gamma}}, \quad i \in \mathbb{N}
$$

где $a_{1}$ и $a_{2}-$ из (6.3). Последовательность $\left\{M_{i}(s)\right\}$ не является монотонной последовательностью, но обладает в силу условий монотонности на последовательности $\left\{k_{i}\right\},\left\{K_{i}\right\},\left\{k_{i} K_{i}^{\gamma}\right\}$ следуюшим важным для нас свойством: сушествует строго монотонно убываюшая последовательность $\left\{s_{i}\right\}$ такая, что $s_{i} \rightarrow 0$ при $i \rightarrow \infty$, а также

$$
\begin{aligned}
& M_{i+1}\left(s_{i}\right)=M_{i}\left(s_{i}\right), \quad i=1,2, \ldots \\
& M_{i+1}(s)>M_{i}(s) \quad \forall s, \quad 0<s<s_{i} \\
& M_{i+1}(s) \leqslant M_{i}(s) \quad \forall s, \quad s_{i}<s<\infty
\end{aligned}
$$

По этой последовательности $\left\{s_{i}\right\}$ определим функцию

$$
U_{0}(s)=M_{i}(s) \quad \forall s \in\left[s_{i}, s_{i-1}\right], \quad i=1,2,3, \ldots ; s_{0}=+\infty .
$$

Докажем, что эта функция является искомой, т.е. выполняется априорная оценка (6.14). Очевидно неравенство:

$$
M_{i}(s) \leqslant U_{0}(s) \quad \forall s>0, \quad \forall i \in \mathbb{N}
$$

Введем еще монотонное семейство монотонно невозрастающих функций:

$$
U_{0}^{(i)}(s)=\left\{\begin{array}{l}
M_{i}(s) \text { при } s \in\left[0, s_{i-1}\right] \\
M_{j}(s) \text { при } s \in\left[s_{j}, s_{j-1}\right] \forall j \leqslant i-1 .
\end{array}\right.
$$

Очевидно, что

$$
U_{0}^{(i)}(s)=U_{0}(s) \quad \forall s \geqslant s_{i}, \quad U_{0}^{(i)}(s)<U_{0}(s) \quad \forall s<s_{i}, \quad \forall i \in \mathbb{N} .
$$

Мы будем доказывать неравенство:

$$
U_{i}(s) \leqslant U_{0}^{(i)}(s) \quad \forall i \in \mathbb{N}, \quad \forall s \in(0, \infty),
$$

которое в силу соотношений (6.22) приведет к требуемой априорной оценке (6.14). При $i=1$ неравенство (6.23) очевидно. Предположим, что это неравенство имеет 
место при всех $i \leqslant j-1$. Докажем, что оно имеет место и при $i=j$. Доказываем от противного. Предположим, что сушествует интервал $\Delta=(v, w)$ такой, что

$$
\begin{gathered}
U_{j}(s)>U_{0}^{(j)}(s) \quad \forall s \in \triangle ; \\
U_{j}(v)=U_{0}^{(j)}(v), \quad U_{j}(w)=U_{0}^{(j)}(w) .
\end{gathered}
$$

В силу (6.11), (6.12) имеем также:

$$
U_{j}(s) \leqslant a+\lambda U_{j-1}(s)+k_{j}\left(-U_{j}^{\prime}(s)\right)^{1+\gamma} \quad \forall s \in(0, \infty) ; \quad U_{j}(0) \leqslant K_{j} .
$$

В силу (6.18) имеем:

$$
U_{0}^{(j)}(s)=M_{j}(s)>M_{j-1}(s) \quad \forall s \in \triangle_{j-1} \equiv \triangle \cap\left(0, s_{j-1}\right) .
$$

По предположению же индукции имеем также:

$$
U_{j-1}(s) \leqslant M_{j-1}(s) \equiv U_{0}^{(j-1)}(s) \quad \forall s \in \triangle_{j-1} .
$$

Теперь в силу $(6.24),(6.27),(6.28)$ получаем из соотношения (6.26):

$$
U_{j}(s) \leqslant a+\lambda U_{j}(s)+k_{j}\left(-U_{j}^{\prime}(s)\right)^{1+\gamma} \quad \forall s \in \triangle_{j},
$$

а в силу $(6.25)$

$$
U_{j}(v)=U_{0}^{(j)}(v)=M_{j}(v) .
$$

Интегрируя теперь дифференциальное неравенство (6.29), получим с учетом (6.30)

$$
U_{j}(s) \leqslant M_{j}(s)=U_{0}^{(j)}(s) \quad \forall s \in \triangle_{j},
$$

откуда ввиду предположения (6.24) следует, что $\triangle \cap\left(0, s_{j-1}\right)=\varnothing$. Пусть теперь

$$
\triangle_{j-2} \equiv \triangle \cap\left[s_{j-1}, s_{j-2}\right) \neq \varnothing, \quad v \in\left[s_{j-1}, s_{j-2}\right) .
$$

По построению функции $U_{0}^{(j)}(s)$ имеем:

$$
U_{0}^{(j)}(s)=M_{j-1}(s) \quad \forall s \in\left[s_{j-1}, s_{j-2}\right),
$$

а из предположения индукции следует:

$$
U_{j-1}(s) \leqslant U_{0}^{(j-1)}(s)=M_{j-1}(s) \quad \forall s \in\left[s_{j-1}, s_{j-2}\right) .
$$

Используя теперь последние соотношения, выводим из неравенства (6.24):

$$
U_{j}(s)>U_{j-1}(s) \quad \forall s \in \triangle_{j-2} .
$$

Поэтому из (6.26) с учетом того, что $k_{j} \leqslant k_{j-1}$, выводим неравенство:

$$
U_{j}(s) \leqslant a+\lambda U_{j}(s)+k_{j-1}\left(-U_{j}^{\prime}(s)\right)^{1+\gamma} \quad \forall s \in \triangle_{j-2},
$$

причем в силу $(6.25)$ и $(6.31)$

$$
U_{j}(v)=U_{0}^{(j)}(v)=M_{j-1}(v) .
$$

Интегрируя неравенство (6.32) с учетом (6.33), получаем оценку:

$$
U_{j}(s) \leqslant M_{j-1}(s)=U_{0}^{(j)}(s) \quad \forall s \in \triangle_{j-2} .
$$

Эта оценка с учетом предположения (6.24) доказьвает, что $\triangle_{j-2}=\varnothing$. Продолжая дальше тем же способом, устанавливаем, что

$$
\triangle \cap\left[s_{i}, s_{i-1}\right)=\varnothing \quad \forall i \leqslant j-1 .
$$

Тем самым лемма 6.2 доказана. 
ЛЕмма 6.3. Пусть в условиях леммы 6.2 параметры системы (6.11), (6.12) удовлетворяют дополнительному соотношению:

$$
K_{i}=r k_{i}^{\frac{-1+\mu}{\gamma}} \quad \forall i \in \mathbb{N}, \quad 0<\mu=\text { const }<1, \quad r<\infty .
$$

Тогда для всего семейства функиий $\left\{U_{i}(s)\right\}$ справедлива равномерная оцен$\kappa a(6.14) c$

$$
U_{0}(s)=c\left(s^{\frac{-1+\mu}{\mu} \cdot \frac{1+\gamma}{\gamma}}+a\right), \quad c=\text { const }<\infty .
$$

ДокАЗАТЕЛьство проведем для случая $a=0$ (в целях сокращения вычислений). Решения соответствующих модельных задач $(6.15),(6.16)$ сейчас принимают вид:

$$
M_{i}(s)=d^{\frac{\gamma+1}{\gamma}} k_{i}^{-\frac{1}{\gamma}}\left[\frac{r^{\frac{\gamma}{1+\gamma}} k_{i}^{\frac{\mu}{1+\gamma}}}{d}-s\right]_{+}^{\frac{\gamma+1}{\gamma}}, \quad i=1,2, \ldots,
$$

где $d=\frac{\gamma}{\gamma+1}(1-\lambda)^{\frac{1}{1+\gamma}}$. Включим последовательность функций $\left\{M_{i}(s)\right\}$ в семейство, зависящее от непрерьвного параметра $\tau$ :

$$
M_{\tau}(s)=d^{\frac{\gamma+1}{\gamma}} \tau^{-1}\left[\frac{r^{\frac{\gamma}{1+\gamma}} \tau^{\frac{\mu \gamma}{1+\gamma}}}{d}-s\right]_{+}^{\frac{\gamma+1}{\gamma}} \quad \forall \tau \in(0, \infty) .
$$

Найдем огибаюшую семейства кривых $y-M_{\tau}(s)=0$. Для этого, как хорошо известно, нужно исключить параметр $\tau$ из системы:

$$
y-M_{\tau}(s)=0, \quad \frac{\partial}{\partial \tau} M_{\tau}(s)=0 .
$$

В результате после несложных вычислений получим искомую огибающую:

$$
y(s)=\frac{r^{\frac{1}{\mu}} \mu^{\frac{1+\gamma}{\gamma}}(1-\mu)^{\frac{(1+\gamma)(1-\mu)}{\gamma \mu}}}{d^{\frac{(\gamma+1)(1-\mu)}{\gamma \mu}}} s^{-\frac{(1+\gamma)(1-\mu)}{\gamma \mu}} \quad \forall s>0 .
$$

Покажем теперь, что

$$
y(s) \geqslant M_{\tau}(s) \quad \forall \tau>0, \quad \forall s>0 .
$$

Так как $y(s)>0, M_{\tau}(s) \geqslant 0$, то для справедливости (6.35) достаточно показать, что

$$
\varphi(s) \equiv y(s)^{\frac{\gamma}{1+\gamma}}-M_{\tau}(s)^{\frac{\gamma}{1+\gamma}} \geqslant 0 \quad \forall \tau>0, \quad \forall s>0 .
$$

Элементарный анализ показывает, что функция $\varphi(s)$ имеет одну экстремальную точку

$$
s_{c}=d^{-1} \tau^{\frac{\gamma \mu}{1+\gamma}}(1-\mu) r^{\frac{\gamma}{1+\gamma}},
$$

т.е. точку, в которой $\varphi^{\prime}\left(s_{c}\right)=0$ для любого значения параметра $\tau$. Просто проверяем, что $\varphi\left(s_{c}\right)=0 \forall \tau>0$, что, очевидно, доказывает неравенство (6.35). Из неравенства (6.35) следует также и требуемая оценка

$$
M_{i}(s) \leqslant c s^{\frac{-(1-\mu)(1+\gamma)}{\mu \gamma}} \quad \forall i \in \mathbb{N}, \quad \forall s>0
$$

с постоянной

$$
c=\frac{r^{\frac{1}{\mu}} \mu^{\frac{\gamma+1}{\gamma}}(1-\mu)^{\frac{(1+\gamma)(1-\mu)}{\gamma \mu}}(1+\gamma)^{\frac{(\gamma+1)(1-\mu)}{\gamma \mu}}}{\gamma^{\frac{(\gamma+1)(1-\mu)}{\gamma \mu}}(1-\lambda)^{\frac{(1-\mu)}{\gamma \mu}}} .
$$

Лемма 6.3 доказана. 


\section{Список литературы}

1. Олейник O.A., Калашников A. C., Чжсу Юйлинь. Задача Коши и краевые задачи для уравнения типа нестационарной фильтрации // Изв. АН СССР. Сер. матем. 1958. T. 22. № 8. C. $667-704$.

2. Aronson D. G. Regularity properties of flows through porous media // SIAM J. Appl. Math. 1969. V. 17. P. 461-467.

3. Gilding B. H. Continuity of generalized solutions of the Cauchy problem for the porous medium equation // J. London Math. Soc. (2). 1976. V. 13. P. 103-106.

4. Баренблатт Г.И. О некоторых неустановившихся движениях жидкости и газа в пористой среде // Прикладная матем. и мех. 1952. Т. 16. № 2. С. 67-78.

5. Баренблатт Г.И., Вишик М.И. О конечной скорости распространения в задачах нестационарной фильтрации жидкости и газа // Прикладная матем. и мех. 1956. Т. 20. №3. C. 411-417.

6. Лихт M.K. О распространении возмущений в задачах, связанных с вырождающимися квазилинейньми уравнениями параболического типа // Дифференц. уравнения. 1966. T. 2. № 7. C. 953-957.

7. Зельдович Я. Б., Компанееч, А. С. К теории распространения тепла при теплопроводности, зависящей от температуры // Сб. посвящ. 70-летию А.Ф. Иоффе. М.: Наука, 1950. C. $61-72$.

8. Caffarelli L. A., Friedman A. Regularity of the free boundary for the one-dimensional flow of gas in a porous medium // Amer. J. Math. 1979. V. 101. P. 1193-1218.

9. Gilding B. H., Herrero M. A. Localization and blow-up of thermal waves in nonlinear heat condaction // Math. Ann. 1988. V. 282. P. 223-242.

10. Самарский А.А., Галактионов В.А., Курдюмов С. П., Михайлов А. П. Режимыс обострением в задачах для квазилинейных параболических уравнений. М.: Наука, 1987.

11. Галактионов B. А., Самарский $A$. А. Методыпостроения приближенных автомодельных решений нелинейных уравнений теплопроводности. I // Матем. сб. 1982. Т. 118. № 3. C. $291-322$.

12. Галактионов В.А., Курдюмов С.П., Михайлов А.П., Самарский А.А. Асимптотическая стадия режимов с обострением и эффективная локализация тепла в задачах нелинейной теплопроводности // Дифференц. уравнения. 1980. Т. 16. № 7. С. 1196-1204.

13. Cortazar C., Elgueta M. Localization and boundedness of the solutions of the Neumann problem for a filtration equation // Nonlinear Anal. 1989. V. 13. № 1. P. 33-41.

14. Антонцев C. H. О локализации решений нелинейных вырождающихся эллиптических и параболических уравнений // Докл. АН СССР. 1981. Т. 260. №6. С. 1289-1293.

15. Diaz J. I., Veron L. Local vanishing properties of solutions of elliptic and parabolic quasilinear equations // Trans. Amer. Math. Soc. 1985. V. 290. № 2. P. 787-814.

16. Олейник О. А., Иосифьян Г. А. Аналоги принципа Сен-Венана и единственность решений параболических граничных задач в неограниченных областях // УМН. 1976. Т. 31. №6. C. $142-166$.

17. Акулов B. Ф., Шишков $A . E$. Об асимптотических свойствах решений смешанных задач для квазилинейных параболических уравнений в неограниченных областях // Матем. сб. 1991. T. 182. № 8 . C. 1200-1210.

18. Alt H. W., Luckhaus S. Quasilinear elliptic-parabolic differential equations // Math. Z. 1983. V. 183. P. 311-341.

19. Benilan Ph., Wittbold P. On mild and weak solutions of elliptic-parabolic problems // Adv. Differential Equations. 1996. V. 1. №6. P. 1053-1073.

Институт прикладной математики и механики НАН Украины, Донецк; 